J. Phys. IV France 130 (2005) 3-35

(C) EDP Sciences, Les Ulis

DOI: $10.1051 /$ jp4:2005130002

\title{
Que peut-on voir avec des neutrons ? Une introduction pour des non spécialistes
}

\author{
J. Schweizer ${ }^{1}$ \\ ${ }^{1}$ CEA-Grenoble, DRFMC/SPSMS/Magnétisme et Diffraction Neutronique, France
}

\begin{abstract}
Résumé. Le neutron est une particule élémentaire qui a été découverte en 1932 par James Chadwick. Ses caractéristiques principales sont résumées dans le tableau I. Il a été utilisé pour la première fois par Clifford Shull en 1946 comme outil pour des expériences de diffusion. Cette technique s'est depuis constamment développée pour concerner tous les aspects de la matière condensée: physique, chimie, matériaux, biologie. Il s'agit d'un outil tout à fait exceptionnel car le neutron possède des propriétés uniques et particulièrement adaptées pour ces études.
\end{abstract}

\section{Table des matières}

1 Généralités sur les neutrons $\quad 4$

1.1 Les propriétés du neutron $\ldots \ldots \ldots \ldots \ldots \ldots$

1.1.1 Le neutron est électriquement neutre . . . . . . . . . . . . . . . . . 4

1.1.2 Le neutron est une particule, mais en même temps une onde . . . . . . . . . . 4

1.1.3 L'énergie du neutron correspond à l'énergie cinétique de la particule . . . . . . 5

1.1.4 Le neutron porte un $\operatorname{spin} s=1 / 2 \ldots \ldots \ldots \ldots \ldots$

1.2 Ondes et corpuscules, les phénomènes de base. Sections efficaces . . . . . . . . . 6

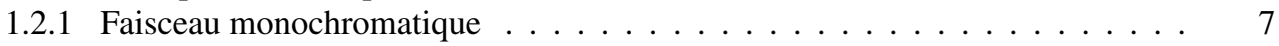

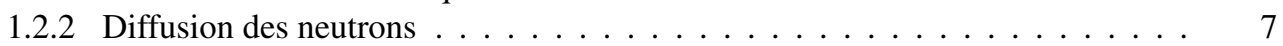

1.2.3 Absorption d'un faisceau de neutrons . . . . . . . . . . . . . . . . . . . . . 8

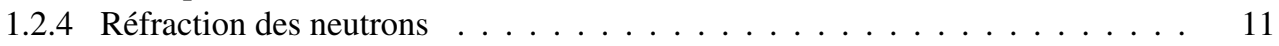

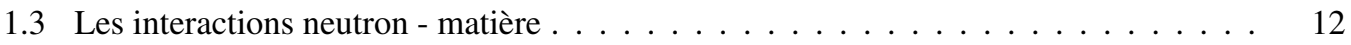

1.3.1 L'interaction nucléaire (neutron - noyau) . . . . . . . . . . . . . . . . . . 12

1.3.2 L'interaction magnétique (pour mémoire) . . . . . . . . . . . . 13

1.4 Production de faisceaux de neutrons $\ldots \ldots \ldots \ldots \ldots$

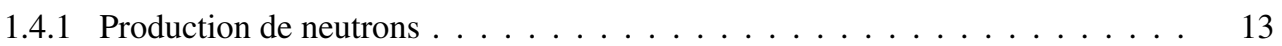

1.4 .2 Modération des neutrons . . . . . . . . . . . . . . . . . . . . . . . . . . 14

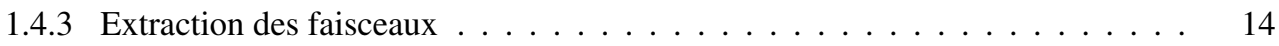

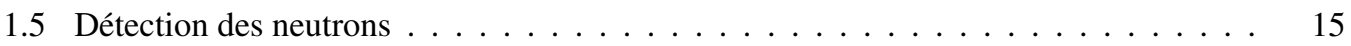

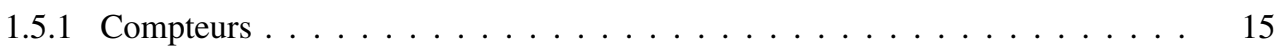

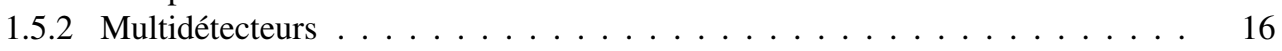

1.5 .3 Imageurs à neutrons $\ldots \ldots \ldots \ldots \ldots \ldots$

2 La diffusion de neutrons mesure les corrélations entre particules dans la matière $\quad 17$

2.1 La diffusion de neutrons : un corréloscope dans l'espace $(\vec{Q}, \omega) \ldots \ldots$. . . . . . . . 17

2.1.1 $\mathrm{G}(\vec{r}, \mathrm{t})$, la fonction de paires dépendant du temps, représente les corrélations dans l'espace $(\vec{r}, \mathrm{t}) \ldots \ldots \ldots \ldots \ldots \ldots$

2.1.2 La fonction intermédiaire $\mathrm{I}(\vec{Q}, \mathrm{t}):$ transformée de Fourier par rapport à $\vec{r}$. . . . 19

2.1.3 Le facteur de structure dynamique $\mathrm{S}(\vec{Q}, \hbar \omega)$ : deuxième transformée de Fourier, cette fois par rapport à t. Section efficace différentielle partielle . . . . 


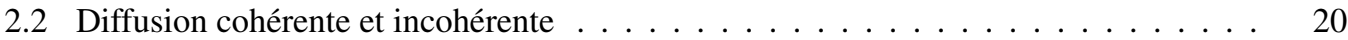

2.2.1 Partage de la section efficace en deux partie . . . . . . . . . . . . . 20

2.2.2 Une autre expression pour la moyenne du carré de l'écart $\overline{(\Delta b)^{2}} \ldots \ldots$. . . . . 21

2.2.3 La limite de $\mathrm{G}(\mathrm{r}, \mathrm{t})$ pour $t \rightarrow \infty$ : Diffusion élastique et diffusion inélastique . $\quad 22$

3 Les instruments de diffusion neutronique $\quad 23$

3.1 Les appareils de diffusion élastique . . . . . . . . . . . . . . . . . . 23

3.1 .1 Les diffractomètres . . . . . . . . . . . . . . . . . . . . . 23

3.1.2 Les appareils de diffusion neutronique à petits angles (DNPA) . . . . . . . . 24

3.1.3 Les spectomètres (diffusion inélastique) . . . . . . . . . . . . . . . . . . 25

3.1 .4 Spectromètre à temps de vol (ou time-of-flight) . . . . . . . . . . . . . . . . . . . 25

3.1 .5 Spectromètre à rétrodiffusion (ou backscattering) . . . . . . . . . . . . . . . 26

3.1.6 Spectromètre à écho de spin (ou neutron spin echo) . . . . . . . . . . . . 27

3.1.7 Les domaines d'utilisation des différents instruments de diffusion inélastique . 27

4 Ce que l'on voit : quelques modèles $\quad 28$

4.1 Voir les structures (diffusion élastique cohérente) . . . . . . . . . . . . . . . . . . 28

4.1 .1 Structure d'un cristal . . . . . . . . . . . . . . . . . . . 29

4.1.2 Structure des objets de grande taille (grosses molécules, grains, précipités...) . 30

4.2 Voir la dynamique à travers les mouvements individuels d'atomes

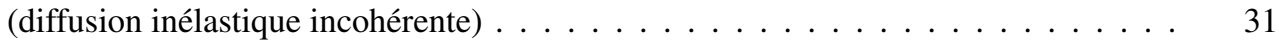

4.2 .1 Les mouvements de translation . . . . . . . . . . . . . . . . . 31

4.2 .2 Les mouvements de rotation . . . . . . . . . . . . . . . . . . 32

4.3 Voir les cages dans lesquelles les atomes restent enfermés

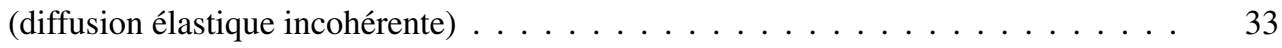

5 Conclusion

\section{GÉNÉRALITÉS SUR LES NEUTRONS}

\subsection{Les propriétés du neutron}

Le neutron est une particule élémentaire qui a été découverte en 1932 par James Chadwick. Ses caractéristiques principales sont résumées dans le tableau I. Il a été utilisé pour la première fois par Clifford Shull en 1946 comme outil pour des expériences de diffusion. Cette technique s'est depuis constamment développée pour concerner tous les aspects de la matière condensée: physique, chimie, matériaux, biologie. Il s'agit d'un outil tout à fait exceptionnel car le neutron possède des propriétés uniques et particulièrement adaptées pour ces études.

\subsubsection{Le neutron est électriquement neutre}

Le neutron possède une charge électrique nulle. De ce fait, il peut pénétrer la matière sans se faire arrêter par une barrière coulombienne. Contrairement aux rayons X qui ne voient, la plupart du temps, que la surface de la matière, les neutrons sondent l'intérieur des échantillons, et c'est là un atout important.

\subsubsection{Le neutron est une particule, mais en même temps une onde}

Le neutron est une particule, mais en même temps une onde, avec une longueur d'onde associée donnée par la formule de de Broglie:

$$
\lambda=\frac{h}{m v}
$$

où h est la constante de Planck, m la masse du neutron et v sa vitesse. 
Table 1. Caractéristiques du neutron, relations et constantes utiles.

\begin{tabular}{|l|l|}
\hline masse & $\mathrm{m}=1.00866 \mathrm{uma}$ \\
& $\mathrm{m}=1.67510^{-27} \mathrm{~kg}$ \\
Spin & $\mathrm{s}=1 / 2$ \\
moment magnétique & $\mu_{n}=9.66310^{-27} \mathrm{~J} . \mathrm{T}^{-1}$ \\
décomposition & $\mu_{n}=1.913 \mu_{B N}$ \\
temps de vie & $\tau=\mathrm{p}+\mathrm{e}^{-}+v$ \\
demi-vie & $\tau=888 \mathrm{~s}$ \\
\hline longueur d'onde & $\mathrm{T}_{1 / 2}=\tau \log (2)=615 \mathrm{~s}$ \\
vecteur d'onde & $\lambda=\frac{h}{m v}$ \\
moment & $\vec{k}\left(k=\frac{2 \pi}{\lambda}\right)$ \\
énergie & $\vec{p}=\hbar \vec{k}$ \\
\hline constantes de Planck & $\mathrm{E}=\frac{1}{2} m v^{2}=\frac{\hbar^{2} k^{2}}{2 m}$ \\
& $\mathrm{~h}=6.62610^{-34} \mathrm{~J} . \mathrm{s}$ \\
magnéton de Bohr & $\hbar=1.05410^{-34} \mathrm{~J}_{\mathrm{s}}$ \\
magnéton de Bohr nucléaire & $1 \mu_{B}=9.27410^{-24} \mathrm{JT}^{-1}$ \\
électron-volt & $1 \mu_{B N}=5.05110^{-27} \mathrm{JT}^{-1}$ \\
\hline
\end{tabular}

Avec des neutrons thermalisés par un modérateur dont la température est $300 \mathrm{~K}$, leur vitesse moyenne est de $2200 \mathrm{~m} / \mathrm{s}$ et leur longueur d'onde moyenne de $1.8 \AA$, c'est à dire une longueur d'onde qui est du même ordre de grandeur que les distances inter atomiques dans la matière condensée. Les neutrons thermiques sont donc tout à fait adaptés pour être diffractés par les atomes de la matière qui nous entoure.

Pour les photons, rappelons le, la longueur d'onde est donnée par:

$$
\lambda=\frac{c}{v}
$$

où $c$ est la vitesse de la lumière et $v$ la fréquence de la radiation. C'est ainsi que ce sont les rayons $\mathrm{X}$, avec des fréquences de $10^{17}$ à $10^{19} \mathrm{~Hz}$ qui correspondent à des longueurs d'onde comparables aux distances entre atomes et molécules.

\subsubsection{L'énergie du neutron correspond à l'énergie cinétique de la particule}

L'énergie du neutron correspond à l'énergie cinétique de la particule et est donnée par la formule classique:

$$
E=\frac{1}{2} m v^{2}=\frac{\hbar^{2} k^{2}}{2 m}
$$

où $k$ est le nombre d'onde $(k=2 \pi / \lambda)$. Cette énergie se retrouve en joules si on exprime la masse en kilogrammes et la vitesse en $\mathrm{m} / \mathrm{s}$. En pratique, on utilise l'électron volt comme unité d'énergie et compte tenu de la formule (1) qui relie la longueur d'onde et la vitesse, on arrive à:

$$
E=\frac{\hbar^{2}}{2 m \lambda^{2}}
$$

ou, à la formule équivalente, avec des unités bien spécifiées:

$$
\lambda(\text { en } \AA) \times \sqrt{E}(\text { E en } e V)=0.286
$$

Ainsi, les neutrons thermiques (longueurs d'onde entre 1 et $5 \AA$ ) ont des énergies dans la gamme des $\mathrm{meV}$, c'est à dire, dans la gamme des excitations de la matière condensée. 


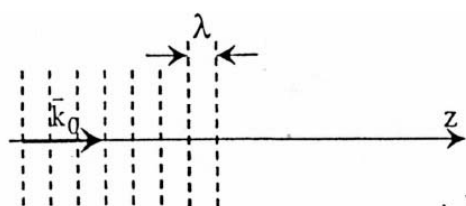

b) Diffusion

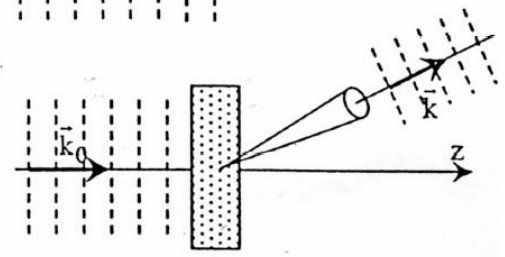

c) Absorption

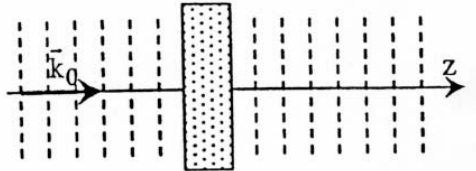

d) Réfraction

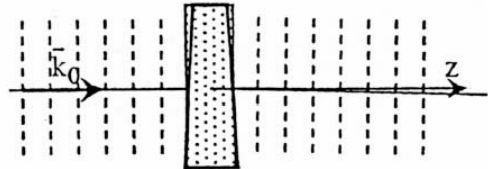

Figure 1. Les phénomènes de base.

Rappelons que pour les photons l'énergie est donnée par:

$$
E=h . v
$$

Ainsi, pour des rayons $\mathrm{X}$ de longueur d'onde $1 \AA ̊$, E vaut $12,4 \mathrm{keV}$, soit 6 ordres de grandeur de plus que les excitations dans la matière condensée. Pour faire de la spectroscopie sur la matière condensée le visible ou l'infrarouge sont de loin préférables aux rayons $\mathrm{X}$. Le neutron est la seule sonde qui permet d'avoir accès en même temps à la structure, par des phénomènes d'interférence, et à la dynamique, par spectroscopie.

\subsubsection{Le neutron porte un spin $\mathrm{s}=1 / 2$}

Un aimant est associé à ce spin. Il est petit et vaut $1.91 \mu_{B N}$ (magnétons de Bohr nucléaires, trois ordres de grandeur plus petit que le magnéton de Bohr électronique). Mais il permet au neutron de ressentir les champs magnétiques dans la matière et, en particulier, ceux qui sont créés par les moments magnétiques électroniques et leurs structures magnétiques. Mais, a côté des études magnétiques, l'existence du spin du neutron joue un grand rôle pour l'étude des objets biologiques. En effet, c'est parce que la diffusion des neutrons par les noyaux d'hydrogène dépend de façon gigantesque des orientations relatives du spin du neutron et du spin du noyau que l'hydrogène joue un rôle tellement particulier. Nous verrons cela en détails plus loin.

\subsection{Ondes et corpuscules, les phénomènes de base. Sections efficaces}

Comment se comporte un faisceau de neutrons dans le vide et dans la matière? Nous allons passer en revue dans ce paragraphe les phénomènes que l'on rencontre. 


\subsubsection{Faisceau monochromatique}

Un faisceau monochromatique de neutrons peut être considéré comme une onde plane. On peut écrire une onde qui se propage selon la direction $\vec{z}$ par la formule:

$$
\Psi=e^{i(k z-2 \pi v t)}
$$

où $v$ est la fréquence de l'onde plane. La photo instantanée d'une telle onde est représentée sur la figure 1a. Les lignes de crête sont figurées par les traits pointillés perpendiculaires à la direction de propagation. Ces lignes de crête sont séparées par une longueur d'onde $\lambda$. Le vecteur d'onde $\vec{k}$ est un vecteur parallèle à la propagation, de longueur $\mathrm{k}=2 \pi / \lambda$ et le moment des neutrons est

$$
\vec{p}=m \vec{v}=\hbar \vec{k}
$$

En tout point de l'espace l'onde est périodique dans le temps, avec une pulsation $\omega$ et une période T données par:

$$
\omega=2 \pi / T=2 \pi \cdot v
$$

\subsubsection{Diffusion des neutrons}

La figure $1 \mathrm{~b}$ représente le phénomène de diffusion. Suite à l'interaction entre le faisceau de neutrons incidents et la matière de l'échantillon, l'échantillon se comporte comme une source et produit une onde diffusée:

$$
\Psi_{d i f}=-f\left(\vec{k}, \overrightarrow{k^{\prime}}\right) \frac{e^{i\left(k^{\prime} r-2 \pi \nu^{\prime} t\right)}}{r}
$$

où l'amplitude de diffusion, $f\left(\vec{k}, \vec{k}^{\prime}\right)$, avec la dimension d'une longueur, caractérise la force de l'interaction neutron-matière. A grande distance de la cible, l'onde diffusée pourra être considérée comme une onde plane, de vecteur d'onde $\vec{k}^{\prime}$. Le vecteur représentant la différence entre les deux vecteurs d'onde est appelé le vecteur de diffusion:

$$
\vec{Q}=\vec{k}-\vec{k}^{\prime}
$$

et le transfert de moment est $\hbar \vec{Q}$.

Si la cible se réduit à une seule particule fixe, cette onde est sphérique et le processus est élastique. Si la cible est plus complexe, il peut y avoir échange d'énergie entre les neutrons et le système diffusant avec $\left|\vec{k}^{\prime}\right| \neq\left|\vec{k}^{\prime}\right|$. Le changement d'énergie du neutron est:

$$
\hbar \omega=E-E^{\prime}=\frac{\hbar^{2}}{2 m}\left(k^{2}-k^{\prime 2}\right)
$$

Par convention $\hbar \omega$ est positif lorsque le neutron cède de l'énergie au système cible.

Ce qui est mesuré, dans une expérience de diffusion, c'est la fraction de neutrons d'énergie incidente $E$, diffusés dans un élément d'angle solide $\mathrm{d} \Omega$, avec une énergie comprise entre $E^{\prime}$ et $E^{\prime}+d E^{\prime}$. C'est la section efficace différentielle partielle qui s'exprime en barns par stéradian et par unité d'énergie:

$$
\frac{d^{2} \sigma}{d \Omega d E^{\prime}}=\frac{1}{\phi_{o}} \frac{\text { nombre de neutrons diffusés par seconde dans } d \Omega \text { et } d E^{\prime}}{d \Omega d E^{\prime}}
$$

où $\phi_{o}$ est le flux de neutrons incidents, c'est à dire le nombre de neutrons par seconde et par unité de surface.

Si on n'analyse pas l'énergie des neutrons diffusés, mais que l'on compte tous les neutrons diffusés dans $d \Omega$, on mesure la section efficace différentielle qui s'exprime en barns par stéradian:

$$
\frac{d \sigma}{d \Omega}=\int d E^{\prime} \frac{d^{2} \sigma}{d \Omega d E^{\prime}}=\frac{1}{\phi_{o}} \frac{\text { nombre de neutrons diffusés par seconde dans } d \Omega}{d \Omega}
$$


Enfin, si on intègre les neutrons diffusés dans toutes les directions de l'espace, on arrive à la section efficace de diffusion totale qui s'exprime en barns:

$$
\sigma_{d i f}=\int d \Omega \frac{d \sigma}{d \Omega}=\frac{\text { nombre de neutrons diffusés par seconde }}{\phi_{o}}
$$

\subsubsection{Absorption d'un faisceau de neutrons}

Absorption vraie. Lorsqu'un faisceau de neutrons traverse de la matière, une partie de ces neutrons peut être absorbée par les noyaux des atomes pour exciter ces noyaux et finalement leur faire éjecter un proton, une particule $\alpha$, un photon $\gamma$ ou même conduire à une fission (figure 1c). Si l'échantillon est une plaque mince où les atomes ne se portent pas ombre les uns sur les autres, le nombre de réactions par seconde $\mathrm{R}$ est proportionnel à l'intensité du faisceau $\mathrm{I}_{o}$ mesuré en neutrons par seconde et à la concentration $\mathrm{n}$ de noyaux sur la plaque (nombre de noyaux par unité de surface):

$$
R(\text { nbre réactions } / \mathrm{s})=I_{o}(\text { nbre neutrons } / \mathrm{s}) \times n\left(\text { nbre noyaux } / \mathrm{cm}^{2}\right) \times \sigma_{a}
$$

La constante de proportionnalité $\sigma_{a}$ a donc les dimensions d'une surface et s'exprime habituellement en barns ( 1 barn $=10^{-24} \mathrm{~cm}^{2}$ ). C'est la section efficace d'absorption qui caractérise la capacité de chaque noyau à absorber les neutrons. Si l'échantillon est maintenant une plaque plus épaisse où il faut tenir compte, pour chaque couche, de l' atténuation du faisceau due à l'absorption par les couches précédentes, l'intensité du faisceau à la sortie de la plaque est donnée par:

$$
I=I_{o} e^{-\mu t}
$$

où $t$ est l'épaisseur de la plaque et où le coefficient d'absorption linéaire $m$ est donné par:

$$
\mu=N . \sigma_{a}
$$

$\mathrm{N}$ représentant cette fois le nombre de noyaux par unité de volume. La section efficace d'absorption est tabulée (voir Tableau 2).

Alors que pour les rayons $\mathrm{X}, \mu$ est de l'ordre de 100 à $1000 \mathrm{~cm}^{-1}(0.01$ à $0.001 \mathrm{~cm}$ de matière suffisent pour affaiblir le faisceau d'un facteur 1/e), la matière est beaucoup plus transparente pour les neutrons car $\mu$ est compris entre 0.01 et $1 \mathrm{~cm}^{-1}$ pour la plupart des matériaux.

L'absorption est un phénomène général des radiations: il dépend de l'énergie et donc de la longueur d'onde des neutrons. On peut le comparer au problème d'un oscillateur amorti et forcé. En effet, il existe des énergies de résonance pour les neutrons qui produisent des excitations du noyau composé (noyau + neutron). Au voisinage de ces énergies de résonance l'absorption est très forte et elle diminue au fur et à mesure qu'on s'en éloigne (figure 2). Pour presque tous les noyaux, ces énergies de résonance se trouvent bien au delà des énergies des neutrons thermiques et la section efficace d'absorption $\sigma_{a}$ est alors proportionnelle à $1 / \mathrm{v}$ ou à $\lambda$. C'est pourquoi les tables d'absorption comme le tableau 2 précisent toujours la vitesse ou la longueur d'onde pour laquelle ces sections efficaces sont données, laissant à l'expérimentateur le soin d'en déterminer la valeur pour la longueur d'onde qu'il utilise.

Absorption apparente. Cependant, il ne faut pas oublier qu'à l'intérieur d'un échantillon, l'intensité du faisceau de neutrons ne diminue pas seulement à cause de l'absorption des neutrons, mais aussi parce que ceux-ci sont diffusés. Pour calculer alors le coefficient d'absorption linéaire apparant $\mu_{a p}$ il faut remplacer la formule 1.18 par l'expression suivante:

$$
\mu_{a p}=N \cdot\left(\sigma_{a}+\sigma\right)
$$


Table 2. Compilation des longueurs de diffusion et des sections efficaces.

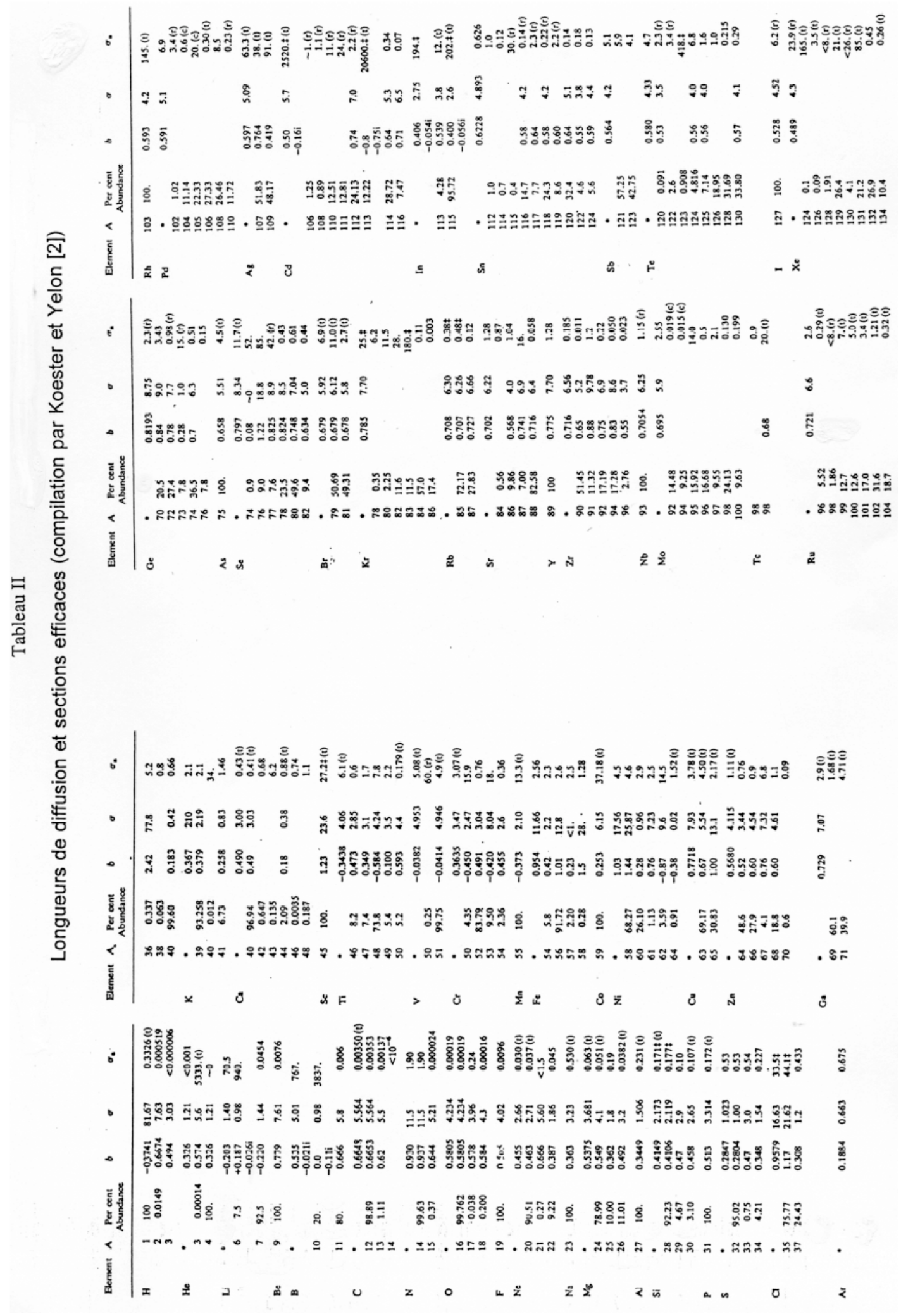


Table 3. (Tab. 2 suite).

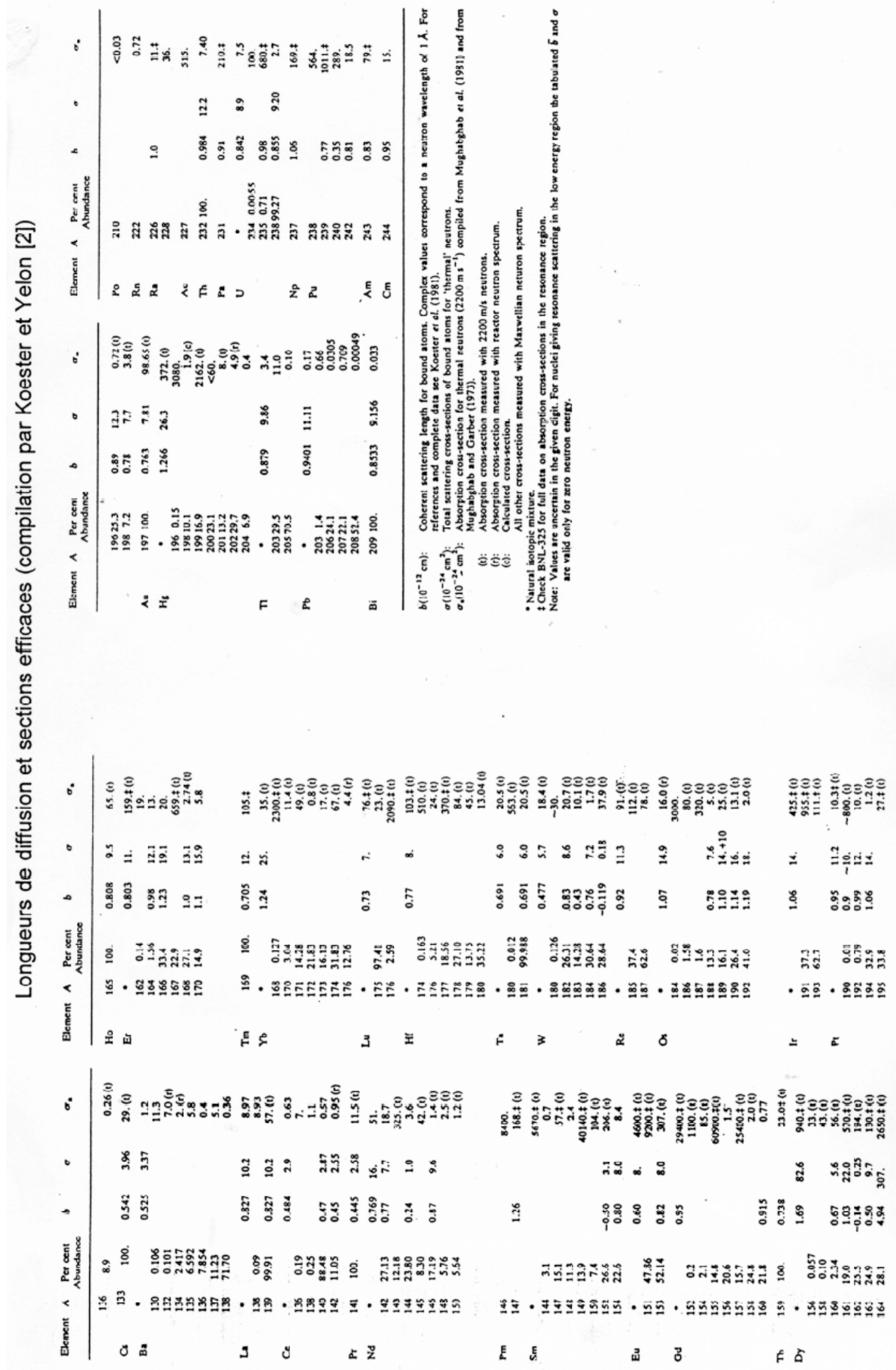




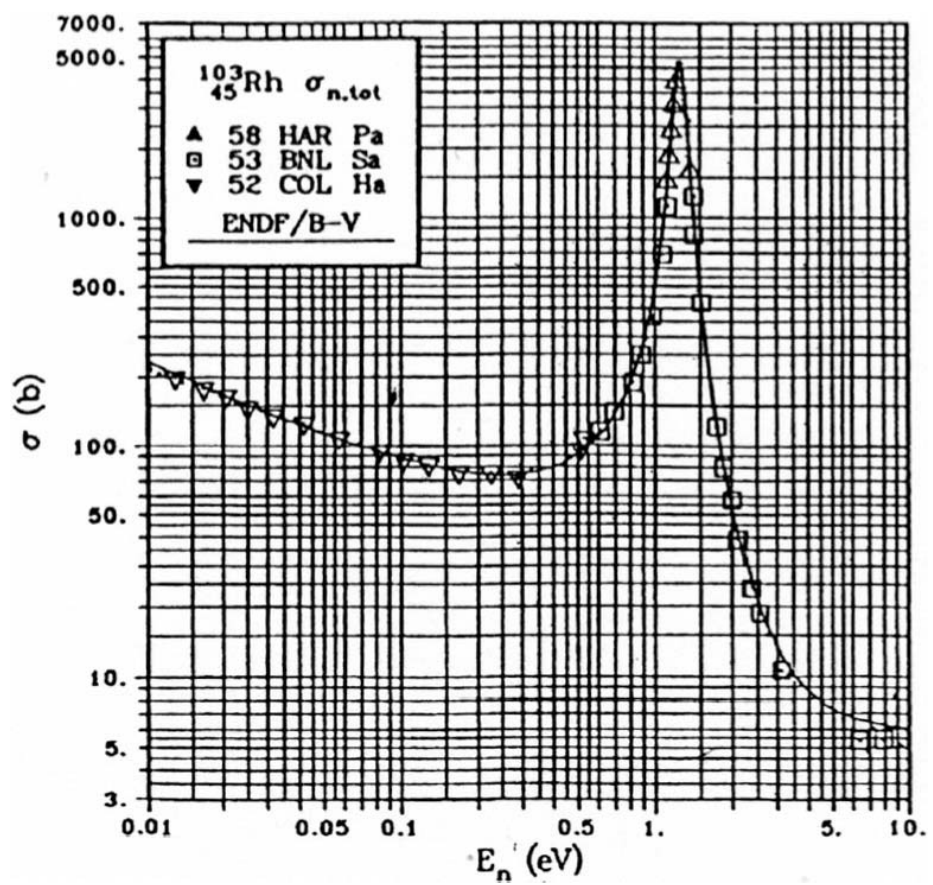

Figure 2. Absorption résonante.

où $\sigma$ est la section efficace de diffusion totale du noyau. La section efficace $\sigma$ est une quantité exprimée en barns qui est indépendante de la longueur d'onde ${ }^{1}$. Elle est également reportée dans le Tableau 2 pour chaque élément, et même pour chaque isotope.

\subsubsection{Réfraction des neutrons}

Le phénomène de réfraction, où le faisceau incident change sa direction au passage à travers un échantillon sans subir une perte importante d'intensité, est bien connu pour la lumière visible à travers une lentille ou un prisme. Il existe aussi pour les neutrons, ainsi d'ailleurs que pour les rayons X. Mais dans ces deux cas les angles de déflexion sont très faibles (voir figure 1d) car, contrairement au cas de la lumière, l'indice de la matière pour les neutrons thermiques (et pour les rayons X) est très voisin de 1 . Le ${ }^{58} \mathrm{Ni}$, un des isotopes les plus réfringents, a un indice de 0.999998 pour une longueur d'onde de 1 A.

Une des manifestations de la réfraction est le phénomène de réflexion totale lorsque les neutrons tombent sur une surface avec un angle d'incidence trop faible. Ils sont alors complètement réfléchis, sans perte d'intensité. Comme les indices sont très voisins de 1 , les angles critiques sont très petits.

On comprend bien ce phénomène de réflexion totale par de simples considérations énergétiques. Le neutron, qui interagit avec la matière par un potentiel $\mathrm{V}(\vec{r})$ verra en fait un potentiel moyen $\mathrm{U}=\langle\mathrm{V}(\vec{r})\rangle$. Son énergie cinétique totale est $\frac{\hbar^{2} k^{2}}{2 m}$ mais la part de cette énergie qui lui permet de l'emporter sur U et de pénétrer dans la matière est $\frac{\hbar^{2} k_{\perp}^{2}}{2 m}$ où $k_{\perp}$ est la composante perpendiculaire à la surface. Si cette énergie est inférieure à $U$, le faisceau de neutrons ne peut pas pénétrer la matière et subit la réflexion totale.

\footnotetext{
${ }^{1}$ Il n'y a que pour les atomes très légers, et en particulier pour l'hydrogène, qu'une variation de $\sigma$ avec l'énergie est à prendre en compte dans le calcul de l'absorption apparente. En effet, selon l'énergie des neutrons, les noyaux légers reculent plus ou moins sous le choc et doivent être considérés comme liés d'une façon plus ou moins rigide au système diffusant. Il s'ensuit alors que la section efficace de diffusion est différente pour un noyau libre ou pour un noyau lié (voir note suivante).
} 
Considérant que $\sin \theta=\frac{k_{\perp}}{k}$, l'angle critique est donné par:

$$
\theta_{c}=\frac{\lambda}{h} \sqrt{2 m U}
$$

Cet angle critique est donc proportionnel à la longueur d'onde. Si, pour $\lambda=1 \AA$, il ne vaut que 7 minutes d'angle dans le cas du ${ }^{58} \mathrm{Ni}$, il monte à plus de 2 degrés lorsque $\lambda$ dépasse $18 \AA$. On utilise ces propriétés de réfraction et de réflexion totale pour mettre en oeuvre toute une optique instrumentale pour les faisceaux de neutrons avec guides, miroirs, multicouches et supermiroirs. Bien entendu, c'est surtout avec des neutrons de grande longueur d'onde, pour des angles critiques les plus grands possibles, que l'optique neutronique est la plus performante.

\subsection{Les interactions neutron - matière}

Il y a deux interactions différentes entre le neutron et les atomes qui forment la matière: l'interaction nucléaire et l'interaction magnétique.

\subsubsection{L'interaction nucléaire (neutron - noyau)}

Il existe des forces nucléaires entre le neutron et le noyau. Ces forces agissent sur des distances très courtes, de l'ordre de grandeur des dimensions du noyau, c'est à dire $10^{-4} \AA$.

La longueur de Fermi. Pour les neutrons utilisés en diffusion, dont la longueur d'onde est comprise entre une fraction d'angström et quelques angströms, on peut représenter le potentiel d'interaction par une fonction $\delta$.

$$
V_{N}(\vec{r})=a . \delta(\vec{r})
$$

On a plutôt coutume de l'écrire sous la forme suivante, dite pseudo potentiel de Fermi:

$$
V_{N}(\vec{r})=\frac{2 \pi \hbar^{2}}{m} b \cdot \delta(\vec{r})
$$

Dans ces conditions, en revenant à la définition (1.10):

$$
f\left(\vec{k}, \vec{k}^{\prime}\right)=\frac{m}{2 \pi \hbar^{2}}\left\langle\overrightarrow{k^{\prime}}\left|V_{N}\right| \vec{k}\right\rangle=b
$$

où $\mathrm{b}$ est la longueur de diffusion ou longueur de Fermi, caractéristique de chaque noyau ${ }^{2}$. Pour un même élément, cette longueur est différente d'un isotope à l'autre.

La diffusion dépendant du spin. En réalité, la situation est plus compliquée que décrite ci-dessus car l'interaction neutron - noyau dépend du spin et cette dépendance intervient lorsque le noyau possède un spin nucléaire $I$.

En fait, dès lors que le noyau possède un $\operatorname{spin}(I \neq 0)$, il y a deux valeurs pour la longueur de diffusion. Selon que le spin total (noyau + neutron) prend la valeur $I+1 / 2$ ou $I-1 / 2$, la longueur de diffusion est $b_{+}$ou $b_{-}$.

Dans la pratique, même si les neutrons sont polarisés, les noyaux, sauf cas exceptionnel, ne le sont pas. Les neutrons sont diffusés au hasard, soit avec une longueur $b_{+}$soit avec une longueur $b_{-}$. Pour calculer la moyenne, il faut prendre en compte le fait que la probabilité pour le spin du noyau composé

\footnotetext{
2 Ces longueurs considèrent que le noyau est fixe car fortement lié au système diffusant. S'il s'agit de noyaux complètement libres, cette longueur de diffusion est plus petite car le noyau, libre de bouger, recule pendant la collision, ce qui donne une longueur de diffusion $\mathrm{b}_{\text {free }}=\mathrm{bM} /(\mathrm{m}+\mathrm{M})$, où $\mathrm{m}$ est la masse du neutron et $\mathrm{M}$ la masse du noyau.
} 
Table 4. Longueurs de diffusion dépendant du spin de quelques noyaux (en $10^{-12} \mathrm{~cm}$ ).

\begin{tabular}{|llllcc|}
\hline noyau & spin & $\overline{\mathrm{b}}$ & $\mathrm{b}_{+}$ & $\mathrm{b}_{-}$ & $\mathrm{b}_{+} \mathrm{b}_{-}$ \\
\hline${ }^{1} \mathrm{H}$ & $1 / 2$ & -0.3741 & 1.085 & -4.750 & 5.835 \\
${ }^{2} \mathrm{H}$ & 1 & 0.6674 & 0.953 & 0.098 & 0.855 \\
${ }^{23} \mathrm{Na}$ & $3 / 2$ & 0.363 & 0.63 & -0.09 & 0.72 \\
${ }^{59} \mathrm{Co}$ & $7 / 2$ & 0.253 & -0.278 & 0.991 & -1.269 \\
\hline
\end{tabular}

de valoir $I+\frac{1}{2}$ est $\frac{I+1}{2 I+1}$ tandis qu'elle est $\frac{I}{2 I+1}$ pour un spin $I-\frac{1}{2}$. D'où:

$$
\overline{\mathrm{b}}=\frac{I+1}{2 I+1} b_{+}+\frac{I}{2 I+1} b_{-}
$$

Le tableau 4 donne la valeur des longueurs $b_{+}$ou $b_{-}$et $\bar{b}$ de quelques isotopes. De tous les atomes, c'est pour le proton $\left({ }^{1} \mathrm{H}\right)$ que la différence entre $b_{+}$ou $b_{-}$est la plus forte. J'insiste sur le fait que, bien que l'interaction neutron - noyau dépende du spin, il s'agit bien d'interaction nucléaire et non pas d'une interaction magnétique avec le moment magnétique du noyau. Cette dernière existe, mais elle est négligeable: par rapport à l'interaction nucléaire ou à l'interaction magnétique avec les moments électroniques. Elle est plus faible de 3 ordres de grandeur.

La moyenne sur les isotopes. Chaque isotope ayant sa propre longueur de diffusion, et comme il n'y a pas de relation entre la nature de l'isotope et sa position, la longueur de diffusion moyenne d'un élément est donnée par une moyenne où interviennent les abondances isotopiques $\mathrm{c}_{\mu}$ :

$$
\overline{\mathrm{b}}=\sum_{\text {isotopes }} c_{\mu} \overline{\mathrm{b}}_{\mu}
$$

La tableau 2, déjà cité pour les sections efficaces d'absorption, donne pour tous les éléments et pour un grand nombre d'isotopes les longueurs de diffusion $\bar{b}$ et $\bar{b}_{\mu}$.

\subsubsection{L'interaction magnétique (pour mémoire)}

Associé à son spin, le neutron porte un moment magnétique $\mu_{n}$ de $1.91 \mu_{B N}$. Ce moment ressent tous les champs magnétiques de la matière et, en particulier, les champs émis par les moments magnétiques $\vec{M}$ des atomes dus aux couches électroniques incomplètes. Grâce à cette interaction on peut étudier les structures magnétiques et la dynamique de ces structures (ondes de spin).

\subsection{Production de faisceaux de neutrons}

\subsubsection{Production de neutrons}

Deux réactions sont utilisées en pratique pour produire des neutrons: la fission et la spallation.

Fission. C'est la réaction qui se produit dans un réacteur (et dans une bombe atomique). Un neutron thermique frappe un noyau ${ }^{235} \mathrm{U}$. Ce noyau devient très excité et se scinde en plusieurs fragments avec émission d'un nombre variable de neutrons: entre 2 et 5, en moyenne 2,5. L'énergie moyenne de ces neutrons est $2 \mathrm{MeV}$.

Spallation. Un proton de haute énergie, issu d'un accélérateur, frappe une cible composée d'atomes lourds (U, W, Hg...). Ces noyaux lourds éjectent alors un certain nombre de particules dont une vingtaine de neutrons ayant une énergie de quelques $\mathrm{MeV}$.

Les jets de protons sur la cible peuvent être pulsés (ISIS) ou continus (SINQ). 

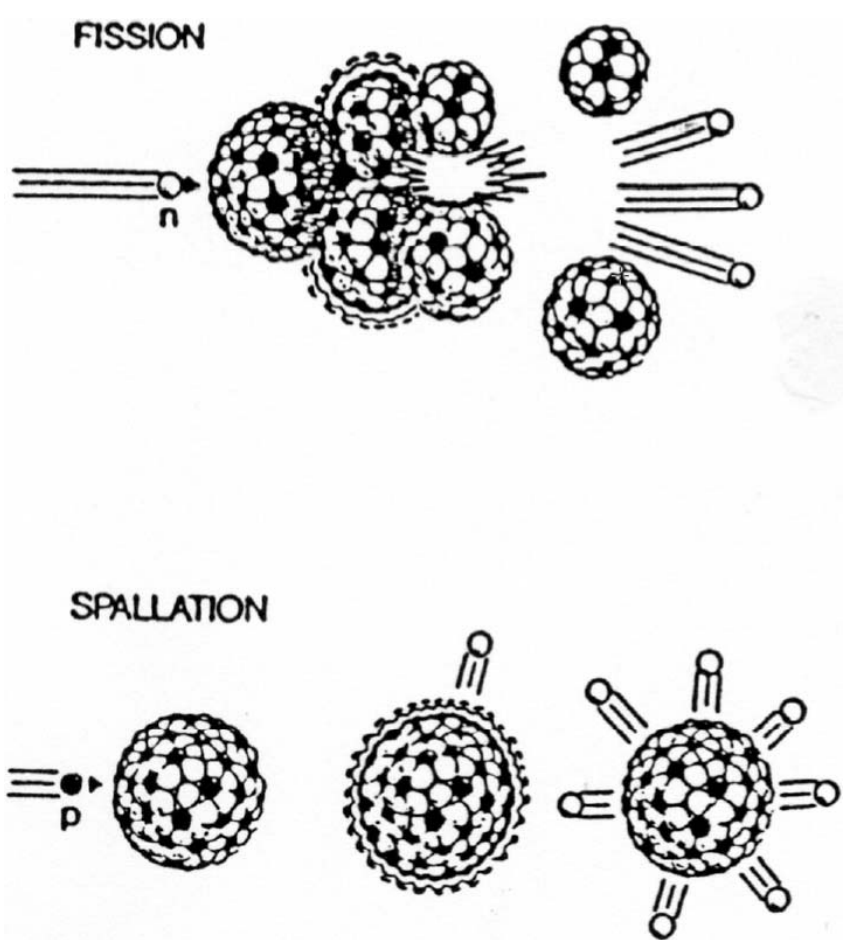

Figure 3. Réactions de fission et de spallation.

\subsubsection{Modération des neutrons}

Les expériences de diffusion neutronique se font avec des neutrons dont l'énergie va d'une fraction de meV à quelques meV. Avec, au départ des neutrons de quelques $\mathrm{MeV}$, il y a 9 ordres de grandeur à perdre pour pouvoir les utiliser. Cette perte d'énergie s'effectue par collisions avec les noyaux d'un modérateur. Plus ces noyaux sont légers, plus le choc est inélastique et plus efficace est le processus de perte d'énergie. Les éléments les plus utilisés pour modérer les neutrons sont l'hydrogène (l'eau), le deutérium (l'eau lourde), le béryllium et le graphite. Après quelques dizaines de collisions, les neutrons sont en équilibre thermique avec le milieu. Il faut pour cela quelques centimètres d'eau ou quelques décimètres d'eau lourde.

Des neutrons en équilibre thermique avec un modérateur à très basse température comme un dewar plein d'hydrogène ou de deutérium liquide (source froide) sont des neutrons de basse énergie (inférieure à $10 \mathrm{meV}$ ) ou neutrons froids. Des neutrons en équilibre thermique avec de l'eau à température ambiante sont dits neutrons thermiques (énergie typique $25 \mathrm{meV}$ ). Des neutrons en équilibre thermique avec un bloc de graphite chauffé à $2000^{\circ} \mathrm{C}$ (source chaude) sont des neutrons chauds (énergie supérieure à $100 \mathrm{meV}$ ). Des spectres caractéristiques des différentes sources sont représentés sur la figure 4.

\subsubsection{Extraction des faisceaux}

On obtient un faisceau de neutrons en ouvrant un canal dans la protection et dans le modérateur, trou par lequel les neutrons bien situés et bien dirigés peuvent s'échapper (voir figure 5). Ces neutrons ont donc la direction imposée par le canal. Leurs vitesses (ou leurs longueurs d'onde) sont réparties selon le spectre maxwellien correspondant à la température du modérateur. 


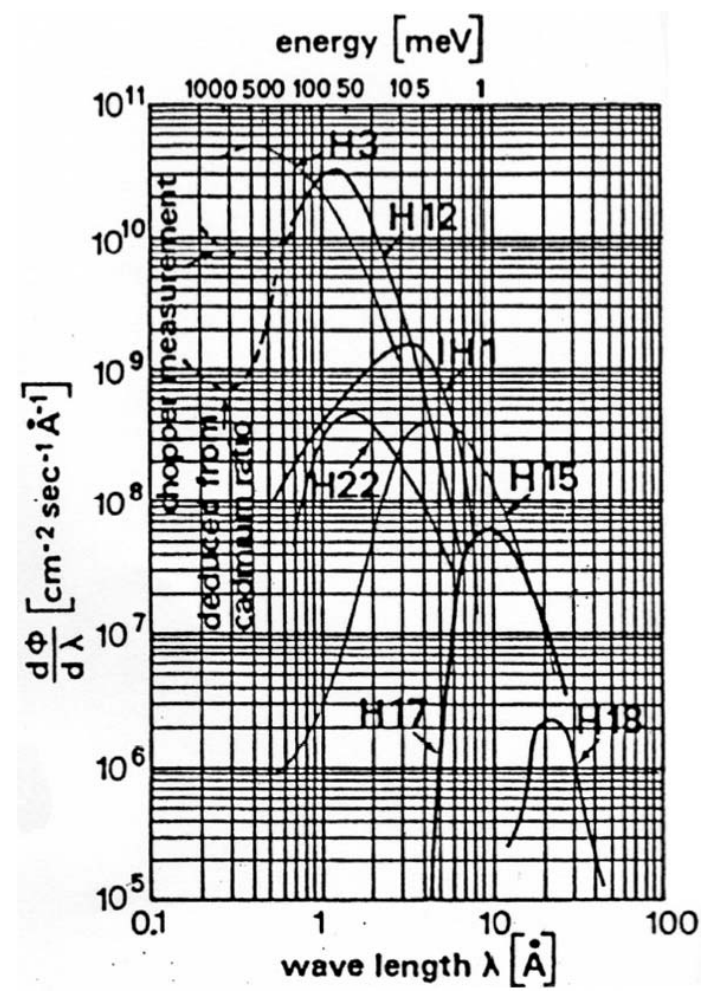

Figure 4. Spectres caractéristiques des différentes sources.

\subsection{Détection des neutrons}

Le neutron n'ayant pas de charge, pour le détecter, il faut lui faire provoquer une réaction nucléaire et détecter soit les particules chargées soit les photons émis par cette réaction.

\subsubsection{Compteurs}

Compteurs proportionnels. Ils sont constitués d'une chambre contenant un gaz absorbant. Il s'agit soit de $\mathrm{BF}_{3}$ enrichi en ${ }^{10} \mathrm{~B}$ :

$$
\mathrm{n}+{ }^{10} \mathrm{~B} \rightarrow{ }^{7} \mathrm{Li}+{ }^{4} \mathrm{He}+\gamma
$$

soit de ${ }^{3} \mathrm{He}$ :

$$
\mathrm{n}+{ }^{3} \mathrm{He} \rightarrow \mathrm{p}+{ }^{3} \mathrm{H}
$$

Les particules chargées émises par la réaction nucléaire ionisent le gaz. Les électrons ainsi émis sont accélérés par les électrodes (voltage de 2 ou $3 \mathrm{kV}$ ) et ionisent encore plus d'atomes de gaz. Il en résulte une décharge sur le fil d'anode. Les photons $\gamma$ créent des impulsions beaucoup plus faibles et faciles à éliminer.

Scintillateurs. Les neutrons sont absorbés dans une couche de verre ou de plastique chargée en ${ }^{6} \mathrm{Li}$ et $\mathrm{ZnS}$.

$$
\mathrm{n}+{ }^{6} \mathrm{Li} \rightarrow{ }^{3} \mathrm{H}+{ }^{4} \mathrm{He}
$$




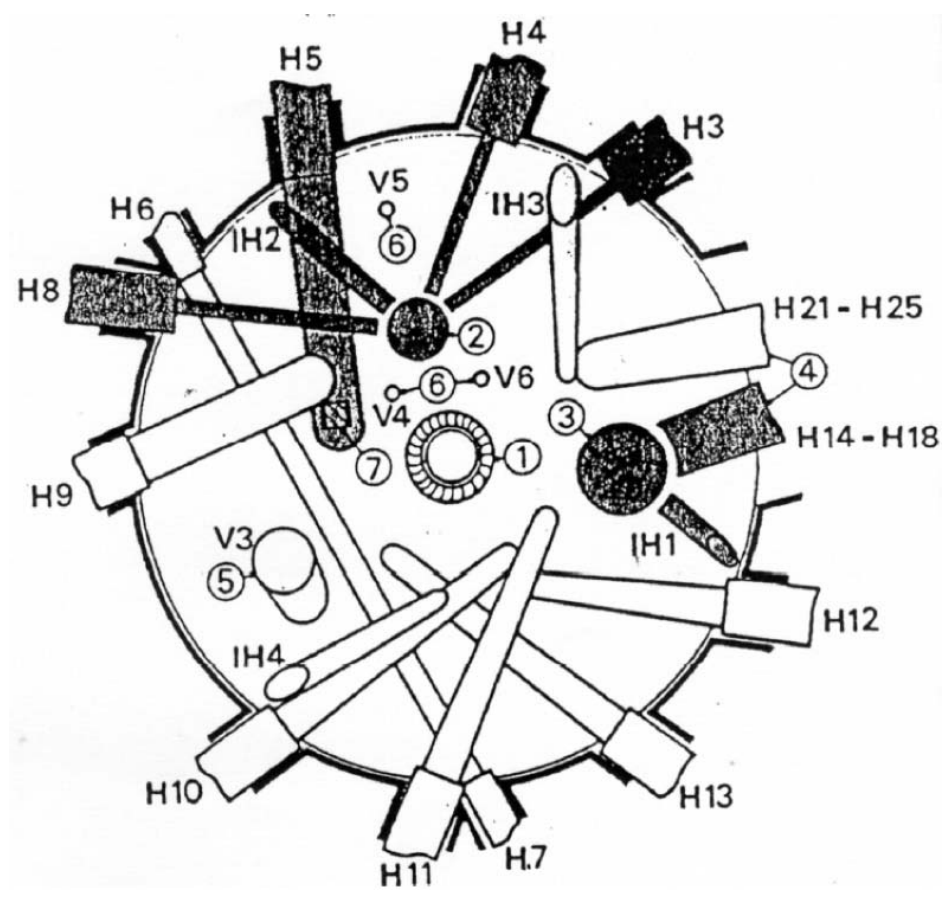

Figure 5. Disposition des sources et des canaux à l'ILL.

Les particules chargées créent des excitations électroniques qui se désactivent en donnant des photons. Ces photons sont transportés par un guide de lumière vers un photomultiplicateur.

Les scintillateurs ont une zone d'absorption des neutrons moins épaisse que les chambres à gaz. Ils sont de ce fait plus précis pour les expériences de temps de vol.

Chambres à fission. Une feuille d'uranium solide est fixée dans une chambre remplie de gaz. Les particules émises dans la réaction de fission sont comptées dans la chambre.

\subsubsection{Multidétecteurs}

Plutôt que de déplacer un compteur unique dans toutes les directions de l'espace pour compter les neutrons diffusés, il est beaucoup plus rentable de tapisser l'espace de surfaces de détection sensibles.

La première possibilité a été d'utiliser un certain nombre de détecteurs individuels. Puis sont apparus les détecteurs sensibles à la position (Position Sensitive Detector ou PSD en anglais) à une ou deux dimensions. A une dimension on peut utiliser un fil résistif qui permet de localiser la position où le neutron a été absorbé (voir figure 6a) ou bien un grand nombre de fils parallèles comme sur la banane de la figure $6 \mathrm{~b}$. A deux dimensions le détecteur implique 2 systèmes de fils, perpendiculaires, qui permettent une localisation en $\mathrm{x}$ et en y (voir figure 7).

L'usage des multidétecteurs s'est généralisé au cours des deux dernières décennies car c'est un moyen d'augmenter l'efficacité d'un instrument de façon beaucoup plus économique que d'augmenter le flux de la source de neutrons. 
(a)

resistive wire gas detector
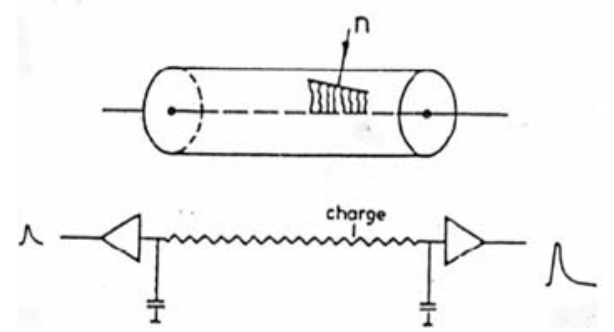

(b)

banana: a multiwire gas detector

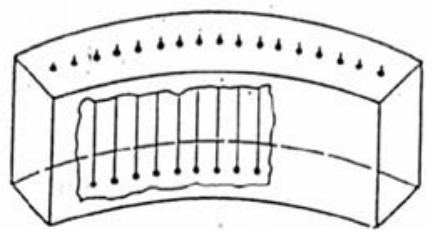

Figure 6. PSD à une dimension: (a) à fil résistif, (b) banane multifils.

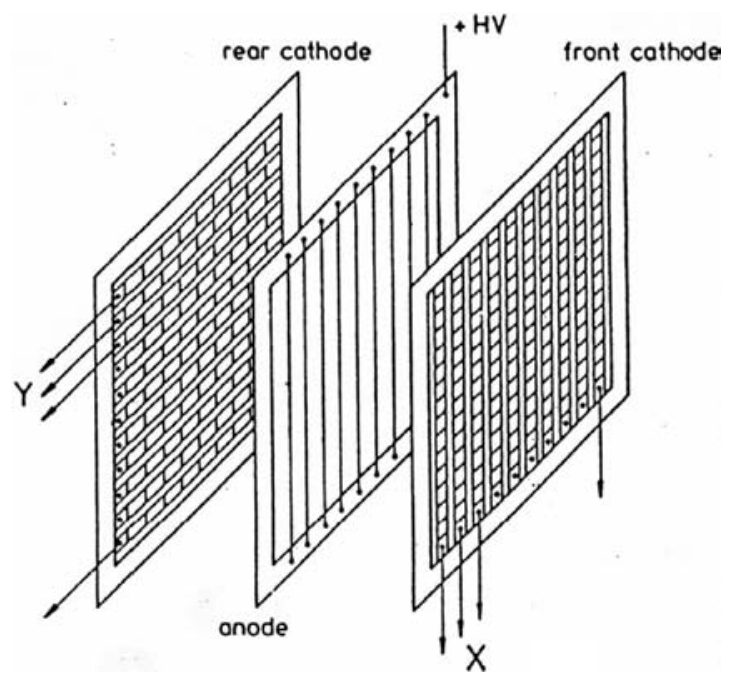

Figure 7. PSD à deux dimensions.

\subsubsection{Imageurs à neutrons}

Au delà des multidétecteurs, mais avec la même finalité de détection sur une surface, se sont développés ces dernières années des imageurs à neutrons (image plates en anglais). Comme pour les rayons X, il s'agit de surfaces contenant du phosphore photostimulable. Mais la spécificité de ces surfaces pour les neutrons est qu'elles contiennent aussi de l'oxyde de gadolinium. Gd joue le rôle de scintillateur: quand il est frappé par un neutron, il donne des rayons $\gamma$ et des électrons de conversion, tous les deux efficaces pour exciter le phosphore. Avec de telles surfaces sensibles, on a là toute la maniabilité du papier photo si répandu en diffusion des rayons $\mathrm{X}$.

\section{LA DIFFUSION DE NEUTRONS MESURE LES CORRÉLATIONS ENTRE PARTICULES DANS LA MATIÈRE}

\subsection{La diffusion de neutrons : un corréloscope dans l'espace $(\overrightarrow{\boldsymbol{Q}}, \omega)$}

Nous allons voir dans cette partie comment les corrélations de particules dans l'espace $(\vec{r}, \mathrm{t})$, l'espace réel où nous vivons, sont mesurées par les neutrons dans l'espace réciproque $(\vec{Q}, \omega)$. 
2.1.1 G( $\vec{r}, t)$, la fonction de paires dépendant du temps, représente les corrélations dans l'espace $(\vec{r}, t)$

Pour comprendre ce qu'est une fonction de corrélation, nous allons donner l'exemple développé par Scherm et Fak [2] sur le "corréloscope à mouches". Des mouches volent dans une pièce qui représente notre espace direct ou espace $(\vec{r}, \mathrm{t})$. Nous voulons les étudier à l'aide d'un corréloscope constitué de 2 viseurs $\mathrm{A}$ et $\mathrm{B}$ reliés par une règle graduée et par un chronomètre (figure 8).

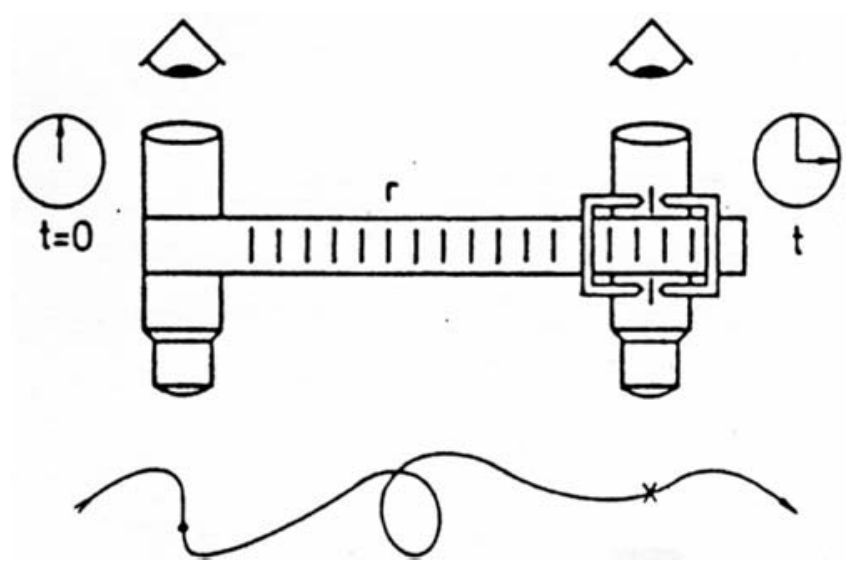

Figure 8. Un corréloscope à mouches, d'après [2].

On commence par chercher une mouche dans le viseur A. Quand on en voit une, on met la règle à zéro en $\mathrm{A}$ et on déclanche le chronomètre. On se met alors à chercher une mouche dans le viseur $\mathrm{B}$. Quand on en voit une, la même ou une autre, on note sa coordonnée d'espace $\vec{r}$ et sa coordonnée de temps t. On recommence l'opération un très grand nombre de fois (d'abord le viseur A, puis le viseur B). On a alors mesuré $P(\rho(\vec{r}, t) \mid \rho(\overrightarrow{0}, 0))$, la probabilité conditionnelle d'avoir une mouche en $(\vec{r}, \mathrm{t})$ si on en a vu une en $(\overrightarrow{0}, 0)$.

C'est cette fonction de l'espace réel que l'on cherche à déterminer par la diffusion de neutrons: la probabilité conditionnelle d'avoir une particule en $(\vec{r}, \mathrm{t})$ si on sait qu'il en existe une en $(\overrightarrow{0}, 0)$. Cette probabilité conditionnelle est appelée fonction de corrélation de paires dépendant du temps $\mathrm{G}(\vec{r}, \mathrm{t})$.

On définit la "densité de particules" $\rho(\vec{r}, \mathrm{t})$, qui est une fonction de l'espace et du temps, de la façon suivante:

$$
\rho(\vec{r}, t)=\sum_{j} \delta(\vec{r}-\vec{R}(t))
$$

La fonction de corrélation de paires dépendante du temps s'écrit alors:

$$
\begin{aligned}
G(\vec{r}, t) & =P(\rho(\vec{r}, t) \mid \rho(\overrightarrow{0}, 0)) \\
& =\frac{1}{N} \int d \overrightarrow{r^{\prime}}\left[\rho\left(\overrightarrow{r^{\prime}}, 0\right) \rho\left(\overrightarrow{r^{\prime}}+\vec{r}, t\right)\right] \\
& =\frac{1}{N} \sum_{j j^{\prime}} \int d \overrightarrow{r^{\prime}}\left[\delta\left(\vec{r}^{\prime}-\vec{R}_{j}(0)\right) \delta\left(\overrightarrow{r^{\prime}}+\vec{r}-\vec{R}_{j^{\prime}}(t)\right)\right]
\end{aligned}
$$

où $\mathrm{N}$ est le nombre de particules. 
Mais les neutrons ne mesurent pas directement cette fonction de corrélation dans l'espace $(\vec{r}, \mathrm{t})$. Ils mesurent une fonction dans l'espace $(\vec{Q}, \omega)$ : la fonction $S(\vec{Q}, \omega)$ qui est la double transformée de Fourier de $\mathrm{G}(\vec{r}, \mathrm{t})$ par rapport à l'espace et au temps.

$$
S(\vec{Q}, \omega)=\operatorname{DFT}(\mathrm{P}[\rho(\vec{r}, t) \mid \rho(\overrightarrow{0}, 0)])=\operatorname{DFT}(\mathrm{G}(\vec{r}, t))
$$

En fait, dans la mesure où toutes les particules ne diffusent pas les neutrons de la même manière, nous devons pondérer les quantités précédentes par les amplitudes de diffusion $b_{j}$ de chaque atome et définir une "densité nucléaire" ou "densité de particules pondérée" $\hat{\rho}(\vec{r}, t)$ au lieu de la "densité de particules" $\rho(\vec{r}, t)$ :

$$
\hat{\rho}(\vec{r}, t)=\sum_{j} b_{j} \delta\left(\vec{r}-\vec{R}_{j}(t)\right)
$$

Et la fonction de corrélation de paires $\mathrm{G}(\vec{r}, \mathrm{t})$ à laquelle on accède par diffusion de neutrons doivent elles aussi être pondérées par les amplitudes $\mathrm{b}_{j}$ :

$$
\begin{aligned}
\hat{G}(\vec{r}, t)=\mathrm{P}[\hat{\rho}(\vec{r}, t) \mid \hat{\rho}(\overrightarrow{0}, 0)] & =\frac{1}{N} \int d \overrightarrow{r^{\prime}}\left[\hat{\rho}\left(\overrightarrow{r^{\prime}}, t\right) \hat{\rho}\left(\overrightarrow{r^{\prime}}+\vec{r}, t\right)\right] \\
& =\frac{1}{N} \sum_{j j^{\prime}} \int d \vec{r}^{\prime} b_{j} b j^{\prime}\left[\delta\left(\overrightarrow{r^{\prime}}-\vec{R}_{j}(0)\right) \delta\left(\overrightarrow{r^{\prime}}+\vec{r}-\vec{R}_{j^{\prime}}\right)\right]
\end{aligned}
$$

\subsubsection{La fonction intermédiaire $I(\vec{Q}, t)$ : transformée de Fourier par rapport à $\vec{r}$}

Si $\mathrm{f}(\vec{r})$ est une fonction de l'espace $\vec{r}$, sa transformée de Fourier $\mathrm{h}(\vec{Q})$ est une fonction de l'espace réciproque $\vec{Q}$ donnée par la relation suivante:

$$
h(\vec{Q})=\int f(\vec{r}) e^{i \vec{Q} \cdot \vec{r}} d^{3} \vec{r}
$$

où l'intégrale est une intégrale triple de l'espace $\vec{r}$.

L'intégrale de Fourier de la fonction de corrélations de paires $\mathrm{G}(\vec{r}, \mathrm{t})$ par rapport à la variable d'espace $\vec{r}$ est la fonction intermédiaire $I(\vec{Q}, t)$, (ou pondérée $\hat{I}(\vec{Q}, t))$.

$$
\begin{aligned}
\hat{I}(\vec{Q}, t) & =\int \hat{G}(\vec{r}, t) e^{i \vec{Q} \cdot \vec{r}} d^{3} \vec{r} \\
& =\frac{1}{N} \sum_{j j^{\prime}} \int d^{3} \vec{r} e^{i \vec{Q} \cdot \vec{r}} \int d \vec{r}^{\prime} b_{j} b_{j^{\prime}} \delta\left(\vec{r}-\vec{R}_{j}(0)\right) \delta\left(\overrightarrow{r^{\prime}}+\vec{r}-\vec{R}_{j^{\prime}}(t)\right)
\end{aligned}
$$

En prenant en compte que

$$
\int \delta(\vec{r}-\vec{R}) e^{i \vec{Q} \cdot \vec{r}} d^{3} \vec{r}=e^{i \vec{Q} \cdot \vec{R}}
$$

on arrive, pour la fonction intermédiaire, à la formule suivante:

$$
\hat{I}(\vec{Q}, t)=\frac{1}{N} \sum_{j j^{\prime}} b_{j} b_{j^{\prime}}\left(e^{-i \vec{Q} \cdot \vec{R}_{j}(0)} e^{i \vec{Q} \cdot \vec{R}_{j^{\prime}}(t)}\right)
$$

Nous verrons plus loin que, contrairement aux autres appareils de diffusion de neutrons qui mesurent la double transformée de Fourier de la fonction de corrélation de paires, les appareils de spin echo mesurent directement la fonction intermédiaire, dans l'espace $(\vec{Q}, \mathrm{t})$. 
2.1.3 Le facteur de structure dynamique $S(\vec{Q}, \hbar \omega)$ : deuxième transformée de Fourier, cette fois par rapport à $t$. Section efficace différentielle partielle

La deuxième transformée de Fourier est une transformée de Fourier par rapport au temps. Dans la mesure où la pulsation $\omega$ est la variable conjuguée du temps t dans les transformées de Fourier, la transformée de Fourier d'une fonction $f(t)$ s'écrit:

$$
g(\omega)=\frac{1}{2 \pi} \int_{-\infty}^{+\infty} f(t) e^{-i \omega t} d t
$$

Dans la mesure où on veut faire apparaître le transfert d'énergie E - E' $=\hbar \omega$ plutôt que la pulsation $\omega$ :

$$
g(\hbar \omega)=\frac{1}{2 \pi} \int_{-\infty}^{+\infty} f(t) e^{-i(\hbar \omega) t} d t=\frac{1}{2 \pi \hbar} \int_{-\infty}^{+\infty} f(t) e^{-i \omega(\hbar t)} d(\hbar t)
$$

On définit alors le facteur de structure dynamique $\mathrm{S}(\vec{Q}, \hbar \omega)^{3}$ ou $\hat{S}(\vec{Q}, \hbar \omega)$ (pondéré par les amplitudes de diffusion atomiques):

$$
\begin{aligned}
\hat{S}(\vec{Q}, \hbar \omega) & =\frac{1}{2 \pi \hbar} \int_{-\infty}^{\infty} \hat{I}(\vec{Q}, t) e^{-i \omega t} d t \\
& =\frac{1}{2 \pi \hbar N} \int_{-\infty}^{\infty} \sum_{j j^{\prime}} b_{j} b_{j^{\prime}}\left(e^{-i \vec{Q} \cdot \vec{R}_{j}(0)} e^{-i \vec{Q} \cdot \vec{R}_{j^{\prime}}(t)}\right) e^{-i \omega t} d t
\end{aligned}
$$

Nous sommes pratiquement arrivés à la section efficace différentielle partielle de diffusion, c'est à dire, la fraction du faisceau incident par unité d'angle solide et unité d'énergie, qui entre dans le compteur. Cette section efficace partielle prend en compte le rapport $\frac{k}{k^{\prime}}$ entre les vitesses après diffusion et avant diffusion de façon à assurer la conservation du flux des neutrons:

$$
\left(\frac{d^{2} \sigma}{d \Omega d E}\right)=\frac{k^{\prime}}{k} N \hat{S}(\vec{Q}, \hbar \omega)
$$

que l'on peut aussi écrire:

$$
\left(\frac{d^{2} \sigma}{d \Omega d E}\right)=\frac{k^{\prime}}{k} \frac{1}{2 \pi \hbar} \int_{-\infty}^{+\infty} \sum_{j j^{\prime}}\left(e^{-i \vec{Q} \cdot \vec{R}_{j}(0)} e^{i \vec{Q} \cdot \vec{R}_{j^{\prime}}(0)}\right) e^{-i \omega t} d t
$$

\subsection{Diffusion cohérente et incohérente}

C'est là une particularité très générale et très importante de la diffusion des neutrons qui est due au fait que les différents atomes d'un même élément diffusent les neutrons avec des longueurs de diffusions qui ne sont pas les mêmes d'un atome à l'autre. Ce phénomène existe pour quelques cas de figure dans la diffusion des rayons $\mathrm{X}$, mais n'a pas le caractère systématique que nous rencontrons ici. En diffusion nucléaire, la différence de longueurs de diffusion provient d'une part de la présence d'isotopes qui ont chacun leur propre longueur de Fermi, et d'autre part, dans le cas d'isotopes ayant un spin nucléaire, de l'existence de deux possibilités: $b_{+}$ou $b_{-}$comme longueur de diffusion.

\subsubsection{Partage de la section efficace en deux partie}

Pour simplifier l'exposé, on va supposer dans ce qui suit que le système est monoatomique et que sa longueur de Fermi est réelle. Comme tous les atomes sont chimiquement équivalents, il n’y a pas de

\footnotetext{
${ }^{3} \mathrm{~S}(\vec{Q}, \hbar \omega)$ est habituellement écrit $\mathrm{S}(\vec{Q}, \omega)$. Il y a là un abus d'écriture dans la mesure où il est sous-entendu que $\omega$ représente le transfert d'énergie.
} 
relation entre la valeur de $\mathrm{b}_{j}$ et sa position $\vec{R}_{j}$ :

$$
\left\langle b_{j} b_{j^{\prime}} e^{-i \vec{Q} \cdot \vec{R}_{j}(0)} e^{-i \vec{Q} \cdot \vec{R}_{j^{\prime}}(t)}\right\rangle=\left\langle b_{j} b_{j^{\prime}}\right\rangle\left\langle e^{-i \vec{Q} \cdot \vec{R}_{j}(0)} e^{-i \vec{Q} \cdot \vec{R}_{j^{\prime}}(t)}\right\rangle
$$

La formule (2.14) s'écrit alors:

$$
\left(\frac{d^{2} \sigma}{d \Omega d E}\right)=\frac{1}{2 \pi \hbar} \frac{k^{\prime}}{k} \sum_{j j^{\prime}}\left\langle b_{j} b_{j^{\prime}}\right\rangle \int_{-\infty}^{+\infty}\left(e^{-i \vec{Q} \cdot \vec{R}_{j}(0)} e^{i \vec{Q} \cdot \vec{R}_{j^{\prime}}(0)}\right) e^{-i \omega t} d t
$$

Intéressons-nous à $\left\langle b_{j} b_{j^{\prime}}\right\rangle$. Pour chaque atome, écrivons que:

$$
b_{j}=\bar{b}+\Delta b_{j}
$$

où $\Delta b_{j}$ représente l'écart à la moyenne, ce qui implique que sa propre moyenne est nulle: $\left\langle\Delta b_{j}\right\rangle=0$. On va devoir évaluer la moyenne des produits $b_{j} b_{j^{\prime}}$ pris sur tous les couples jj':

$$
\begin{aligned}
\left\langle b_{j} b_{j^{\prime}}\right\rangle & =\left\langle\left(b_{j}=\bar{b}+\Delta b_{j}\right)\left(b_{j}=\bar{b}+\Delta b_{j^{\prime}}\right)\right\rangle \\
& =\left\langle\left(\bar{b}^{2}\right)+\bar{b} \Delta b_{j}+\bar{b} \Delta b_{j^{\prime}}+\left(\Delta b_{j}\right)\left(\bar{b} \Delta b_{j^{\prime}}\right)\right\rangle
\end{aligned}
$$

Les moyennes du deuxième et du troisième terme sont nulles compte tenu de (2.17). Quant à la moyenne $\operatorname{sur}\left(\Delta b_{j}\right)\left(\bar{b} \Delta b_{j^{\prime}}\right)$, il faut distinguer:

$$
\begin{aligned}
\left\langle\left(\Delta b_{j}\right)\left(\bar{b} \Delta b_{j^{\prime}}\right)\right\rangle & =0 \quad \text { si } \quad j^{\prime}=j \\
& =\overline{(\Delta b)^{2}} \text { si } \quad j^{\prime} \neq j
\end{aligned}
$$

et la section efficace différentielle partielle se sépare en deux parties:

$$
\begin{aligned}
\left(\frac{d^{2} \sigma}{d \Omega d E^{\prime}}\right)= & \frac{1}{2 \pi \hbar} \frac{k^{\prime}}{k}(\bar{b})^{2} \sum_{j j^{\prime}} \int_{-\infty}^{\infty}\left(e^{-i \vec{Q} \cdot \vec{R}_{j}(0)} e^{i \vec{Q} \cdot \vec{R}_{j^{\prime}}(t)}\right) e^{-i \omega t} d t \\
& +\frac{1}{2 \pi \hbar} \frac{k^{\prime}}{k} \overline{(\Delta b)^{2}} \sum_{j} \int_{-\infty}^{\infty}\left(e^{-i \vec{Q} \cdot \vec{R}_{j}(0)} e^{i \vec{Q} \cdot \vec{R}_{j}(t)}\right) e^{-i \omega t} d t
\end{aligned}
$$

- une partie "cohérente" qui représente l'interaction entre paires d'atomes $\left(\sum_{j j^{\prime}}\right)$, construite avec la moyenne du carré des longueurs de Fermi $(\bar{b})^{2}$

- une partie "incohérente" qui représente l'interaction de chaque atome avec lui-même $\left(\sum_{j}\right)$, construite avec la moyenne du carré de l'écart à la moyenne $\overline{(\Delta b)^{2}}$

\subsubsection{Une autre expression pour la moyenne du carré de l'écart $\overline{(\Delta b)^{2}}$}

$$
\begin{aligned}
\left(\Delta b_{j}\right)^{2} & =\left(b_{j}-\bar{b}\right)^{2}=\left(b_{j}^{2}-2 \bar{b} b_{j}+(\bar{b})^{2}\right) \\
\overline{(\Delta b)^{2}} & =\left(\overline{(b)^{2}}-2 \overline{b b}+(\bar{b})^{2}\right)=\overline{(b)^{2}}-(\bar{b})^{2}
\end{aligned}
$$

Pour chaque élément, on définit:

- sa section efficace totale $\sigma=4 \pi \overline{(b)^{2}}$

- sa section efficace cohérente $\sigma_{c}=4 \pi(\bar{b})^{2}$

- sa section efficace incohérente $\sigma_{i}=4 \pi\left(\overline{(b)^{2}}-(\bar{b})^{2}\right)$

et on a bien:

$$
\sigma=\sigma_{c}+\sigma_{i}
$$


Dans le tableau 2 sont tabulées, pour chaque isotope et pour chaque élément, les longueurs de Fermi moyennes $\bar{b}$ et les sections efficaces totales $\sigma=4 \pi \overline{(b)^{2}}$. On peut en déduire la section efficace cohérente $\sigma_{c}=4 \pi(\bar{b})^{2}$ et la section efficace incohérente $\sigma_{i}=\sigma-\sigma_{c}$.

Pour les systèmes monoatomiques, on peut donc remplacer dans l'expression des sections efficaces différentielles partielles $(\bar{b})^{2}$ par $\frac{\sigma_{c}}{4 \pi}$ et $\overline{(\Delta b)^{2}}$ par $\frac{\sigma_{i}}{4 \pi}$. On a alors:

$$
\left(\frac{d^{2} \sigma}{d \Omega d E^{\prime}}\right)=\left(\frac{d^{2} \sigma}{d \Omega d E^{\prime}}\right)_{c o h}+\left(\frac{d^{2} \sigma}{d \Omega d E^{\prime}}\right)_{i n c}
$$

avec:

$$
\left(\frac{d^{2} \sigma}{d \Omega d E^{\prime}}\right)_{c o h}=\frac{1}{2 \pi \hbar} \frac{k^{\prime}}{k} \frac{\sigma_{c}}{4 \pi} \sum_{j j^{\prime}} \int_{-\infty}^{+\infty}\left(e^{-i \vec{Q} \cdot R_{j}(0)} e^{i \vec{Q} \cdot R_{j^{\prime}}(t)}\right) e^{-i \omega t} d t
$$

et:

$$
\left(\frac{d^{2} \sigma}{d \Omega d E^{\prime}}\right)_{i n c}=\frac{1}{2 \pi \hbar} \frac{k^{\prime}}{k} \frac{\sigma_{i}}{4 \pi} \sum_{j} \int_{-\infty}^{+\infty}\left(e^{-i \vec{Q} \cdot R_{j}(0)} e^{i \vec{Q} \cdot R_{j}(t)}\right) e^{-i \omega t} d t
$$

Au cours d'une expérience, on mesure la somme (2.23) de la diffusion cohérente et de la diffusion incohérente. Chacun des termes de cette somme est d'autant plus important que la section efficace $\sigma_{c}$ ou $\sigma_{i}$ des atomes qui entrent en jeu sont importants.

Par exemple, dans une expérience sur un échantillon hydrogéné, la section efficace de l'hydrogène est tellement forte ( $\sigma_{c}=1.8$ barns, $\sigma_{i}=79.9$ barns), qu'elle domine et écrase la diffusion cohérente. Par contre, avec du deutérium $\left(\sigma_{c}=5.6\right.$ barns, $\sigma_{i}=2.0$ barns), la diffusion cohérente domine, mais de façon plus équilibrée.

\subsubsection{La limite de $G(r, t)$ pour $t \rightarrow \infty$ : Diffusion élastique et diffusion inélastique}

Pour tous les systèmes, on peut décomposer la fonction de corrélation de paires dépendant du temps de la façon suivante:

$$
G(\vec{r}, t)=G(\vec{r}, \infty)+G^{\prime}(\vec{r}, t) \text { avec } \lim _{t \rightarrow \infty} G^{\prime}(\vec{r}, t)=0
$$

De même, on peut décomposer la fonction intermédiaire:

$$
I(\vec{Q}, t)=I(\vec{Q}, \infty)+I^{\prime}(\vec{Q}, t) \text { avec } \lim _{t \rightarrow \infty} I^{\prime}(\vec{Q}, t)=0
$$

Ce qui nous donne pour le facteur de structure dynamique:

$$
\begin{aligned}
S(\vec{Q}, \omega) & =\frac{1}{2 \pi \hbar} \int_{-\infty}^{+\infty}\left[I(\vec{Q}, \infty)+I^{\prime}(\vec{Q}, t)\right] e^{-i \omega t} d t \\
& =\frac{\delta(\omega)}{\hbar} I(\vec{Q}, \infty)+\frac{1}{2 \pi \hbar} I^{\prime}(\vec{Q}, t) e^{-i \omega t} d t \\
& =S^{e l}(\vec{Q}, \omega)+S^{i n e l}(\vec{Q}, \omega)
\end{aligned}
$$

Ce qui donne pour la section efficace différentielle partielle:

$$
\left(\frac{d^{2} \sigma}{d \Omega d E^{\prime}}\right)=\left(\frac{d^{2} \sigma}{d \Omega d E^{\prime}}\right)^{e l}+\left(\frac{d^{2} \sigma}{d \Omega d E^{\prime}}\right)^{i n e l}
$$

En notant que pour la diffusion élastique $k^{\prime}=k$, on obtient pour la partie élastique de la diffusion cohérente:

$$
\left(\frac{d \sigma}{d \Omega}\right)_{c o h}^{e l}=\sum_{j j^{\prime}}\left(\bar{b}_{j} \bar{b}_{j^{\prime}} e^{-i \vec{Q} \cdot\left[\vec{R}_{j}(0)-\vec{R}_{j^{\prime}}(\infty)\right]}\right)
$$


et pour la partie élastique de la diffusion incohérente:

$$
\left(\frac{d \sigma}{d \Omega}\right)_{i n c}^{e l}=\sum_{j}\left(\bar{b}_{j} \bar{b}_{j} e^{\left.-i \vec{Q}_{\cdot} \cdot \vec{R}_{j}(0)-\vec{R}_{j}(\infty)\right]}\right)
$$

On voit donc que la diffusion élastique représente:

- Pour la partie cohérente, les interférences entre paires d'atomes: un atome au temps zero et un autre atome au temps infini.

- Pour la partie incohérente, les interférences entre un atome au temps zero et ce même atome au temps infini.

Nous verrons dans la quatrième partie de ce cours comment relier de telles mesures à la structure ou à la dynamique des grosses molécules.

\section{LES INSTRUMENTS DE DIFFUSION NEUTRONIQUE}

\subsection{Les appareils de diffusion élastique}

Pour l'étude des structures, on n'est intéressé que par la fonction $\mathrm{S}(\vec{Q})$. Les instruments utilisés négligent la dépendence en $\omega$ et considèrent que toute la diffusion est élastique.

\subsubsection{Les diffractomètres}

La diffusion élastique par la matière cristallisée est le plus souvent appelée "diffraction" et les appareils correspondants sont les "diffractomètres". Chaque diffractomètre est caractérisé par le domaine des vecteurs $\vec{Q}$ qui sont accessibles sur cet instrument. En effet, plus on mesure loin dans l'espace des $\vec{Q}$ et plus on peut voir des petits détails dans l'espace des $\vec{r}$, donc meilleure est la résolution:

$$
\text { Domaine de résolution } \sim(\text { Domaine de } Q)^{-1}
$$

Les diffractomètres utilisent en général un faisceau incident monochromatique. Ce faisceau est obtenu par réflexion de Bragg sur une série de plans réflecteurs (hkl) d'un gros cristal appelé monochromateur:

$$
2 d_{h k l} \sin \theta=\lambda
$$

Ce monochromateur sélectionne une longueur d'onde unique (un seul $\vec{k}$ ) pour le faisceau tombant sur l'échantillon. Un détecteur mesure l'intensité (section efficace différentielle) diffusée dans la direction $\vec{k}^{\prime}$ où il se trouve. Le détecteur bouge et explore les différents $\vec{k}^{\prime}$ (figure 9). En diffraction classique, on distingue:

- les diffractomètres à poudres

- les diffractomètres à monocristaux

- avec cercle d'Euler (4 cercles)

. à bras levant

L'apparition des imageurs à neutrons a permis de mettre en oeuvre la technique de Laue (ou quasi-Laue), pour étudier la structure d'un cristal. Le faisceau qui tombe sur l'échantillon est polychromatique. Il inclut tout le spectre de neutrons (ou une partie du spectre seulement). L'échantillon est immobile et chaque plan réflecteur sélectionne dans le spectre incident la longueur d'onde qui satisfait la loi de Bragg. Les rayons réfléchis vont impressionner la surface sensible de la même façon que les rayons $\mathrm{X}$ impressionnent un film photographique. Cette technique, utilisée au départ pour orienter des cristaux, s'est avérée très adaptée à la cristallographie des grosses molécules comme les protéines (figure 10). 




Figure 9. Diffractomètre à faisceau monochromatique.



Schematic diagram of detector. 1: Image plate on drum. 2: Drum. 3: Sample holder. 4: Crystal. 5: Transmission belt to drive drum. Motor is under table. 6: Carrier for reading head with photomultiplier. 7: He-Ne-laser. 8: Mirrors for bringing the laser light to the reader head. 9: Reader head with photomultiplier. 10: Encoder for drum rotation. 11: Cover.

Figure 10. Schéma d'un imageur de neutrons avec son système de lecture.

\subsubsection{Les appareils de diffusion neutronique à petits angles (DNPA)}

La diffusion neutronique dite à petits angles (DNPA) est en fait une diffusion à petits vecteurs $Q=\frac{\sin \theta}{\lambda}$. Cette technique utilise en général des neutrons de grande longueur d'onde. Le monochromateur est remplacé par un sélecteur de vitesse mécanique et le détecteur est un multidétecteur (figure 11). 




Figure 11. Diffractomètre de diffusion à petits angles.

\subsubsection{Les spectomètres (diffusion inélastique)}

Ces appareils permettent de connaître l'énergie (ou la vitesse) des neutrons avant et après le processus de diffusion. Chaque appareil, en plus de son domaine de résolution spatiale (domaine en Q), mesure le transfert d'énergie dans un domaine d'énergie $\hbar \omega$ donné. Les dynamiques qui peuvent être étudiées correspondent alors à l'inverse du domaine de pulsation $\omega$. Si le transfert d'énergie $\hbar \omega$ est exprimé en mev, le domaine des temps (en secondes) est donné par:

$$
(\text { Domaine des temps })_{s e c}=(\text { Domaine des } \omega)^{-1}=10^{-12}\left(\text { Domaine des } \hbar \omega_{m e V}\right)^{-1}
$$

\subsubsection{Spectromètre à temps de vol (ou time-of-flight)}

Sur un spectromètre à temps de vol, le faisceau incident est haché et rendu monochromatique par un chopper. De nombreux détecteurs sont installés tout autour de l'échantillon et l'énergie des neutrons diffusés est déduite du temps qu'ils mettent pour parcourir la distance entre échantillon et détecteur.

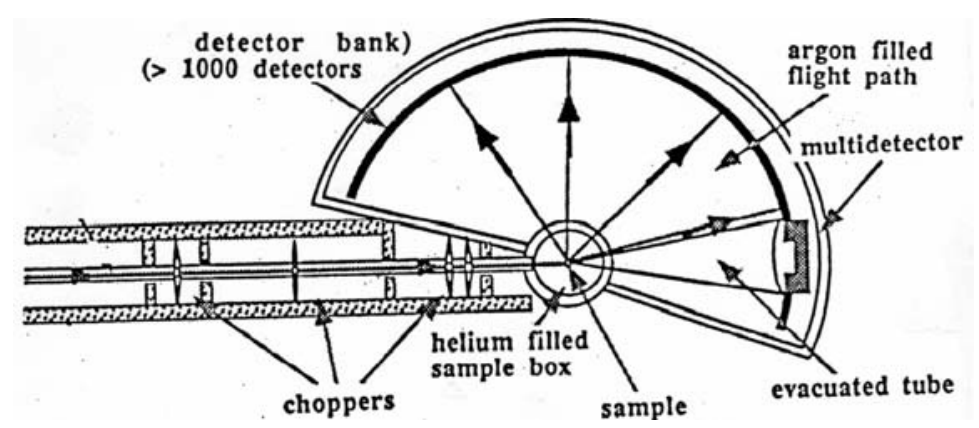

Figure 12. Schéma d'un spectromètre à temps de vol.

Les figures 12 et 13 représentent les schémas de principe du spectromètre à temps de vol IN5 de l'ILL. Le chopper est un multichopper: le premier disque hache le faisceau tandis que les autres disques, selon leur vitesse de rotation, sélectionnent la longueur d'onde des neutrons incidents. Les neutrons arrivent donc tous en même temps sur l'échantillon.

Après diffusion, en fonction de l'énergie que les neutrons ont acquise ou perdue, ils mettent plus ou moins de temps pour atteindre les détecteurs. Ces détecteurs enregistrent, pour chaque neutron reçu, le temps qu'il a mis depuis son passage par le chopper, donc quelle était sa vitesse après diffusion. Il y là mesure du vecteur de diffusion $Q$ en même temps que mesure du transfert d'énergie $\hbar \omega$. Selon les instruments, les spectromètres à temps de vol mesurent des transferts d'énergie qui vont de 10 à $10^{-2}$ mev, et peuvent donc voir des mouvements qui se font entre $10^{-10}$ et $10^{-13}$ secondes. 


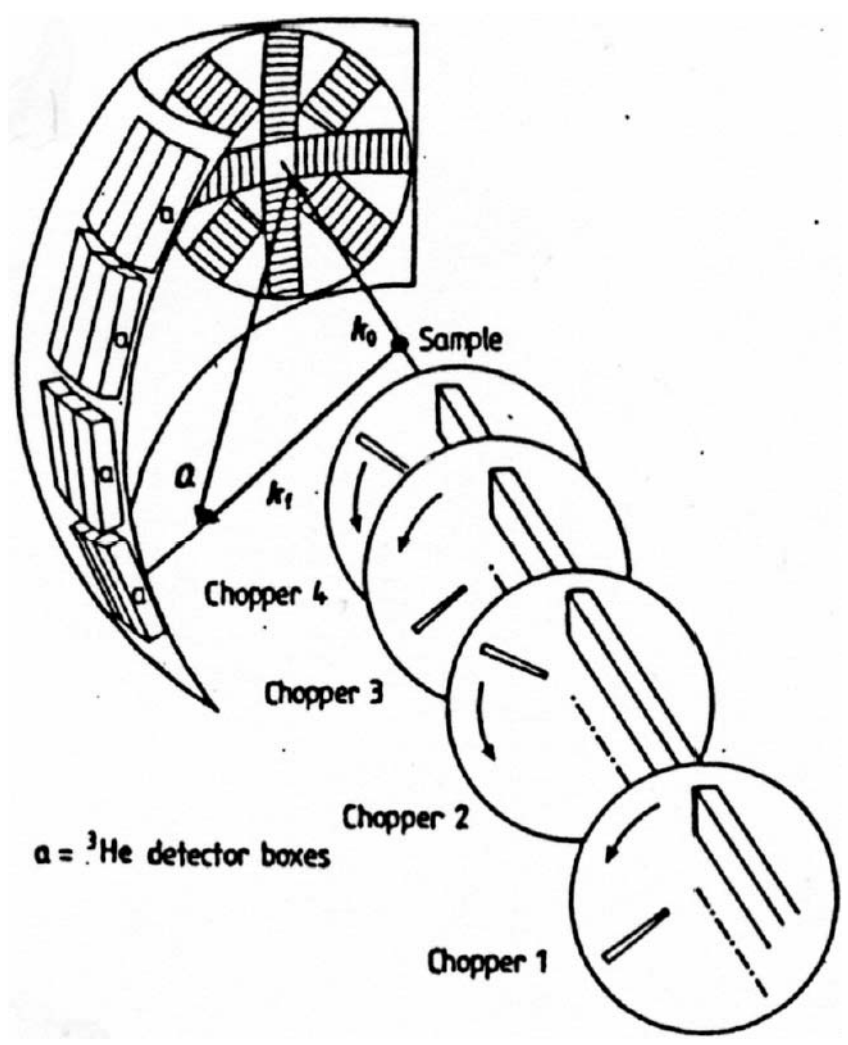

Figure 13. Multichopper du spectromètre à temps de vol IN5 d'après [3].

\subsubsection{Spectromètre à rétrodiffusion (ou backscattering)}

Ce sont des appareils qui comportent un monochromateur, un échantillon et un analyseur, mais où monochromateur et analyseur sont des cristaux parfaits fonctionnant en rétrodiffusion $\left(2 \theta=180^{\circ}\right)$. En différenciant la loi de Bragg pour obtenir la résolution en longueur d'onde:

$$
\left(\frac{\Delta \lambda}{\lambda}\right)^{2}=\left(\frac{\Delta d}{d}\right)^{2}+\left(\frac{\Delta \theta}{\tan \theta}\right)^{2}
$$

on voit que le deuxième terme disparait pratiquement pour $\theta=90^{\circ}$ et la résolution ne dépend plus que du premier terme, c'est à dire de la perfection du cristal (monochromateur ou analyseur).

Les neutrons rétrodiffractés par le monochromateur reviennent sur leurs pas et, grâce à un déflecteur (un cristal à large bande passante), sont envoyés sur l'échantillon. Après diffusion par l'échantillon, les neutrons sont rétrodiffractés par l'analyseur, retraversent l'échantillon et tombent sur un détecteur. En fait il y a un grand nombre d'analyseurs et de détecteurs (figure 14). Un chopper permet d'éliminer les neutrons qui iraient directement de l'échantillon au détecteur, sans passer par l'analyseur. L'exploration en énergie se fait par balayage de la longueur d'onde incidente

- soit par effet Doppler, en imprimant un mouvement au monochromateur

- soit par chauffage du monochromateur, ce qui entraîne une variation continue du paramètre d. On peut ainsi atteindre des résolutions meilleures que le $\mu \mathrm{eV}$, et étudier des mouvements qui se font entre $10^{-12}$ et $10^{-9}$ secondes. 


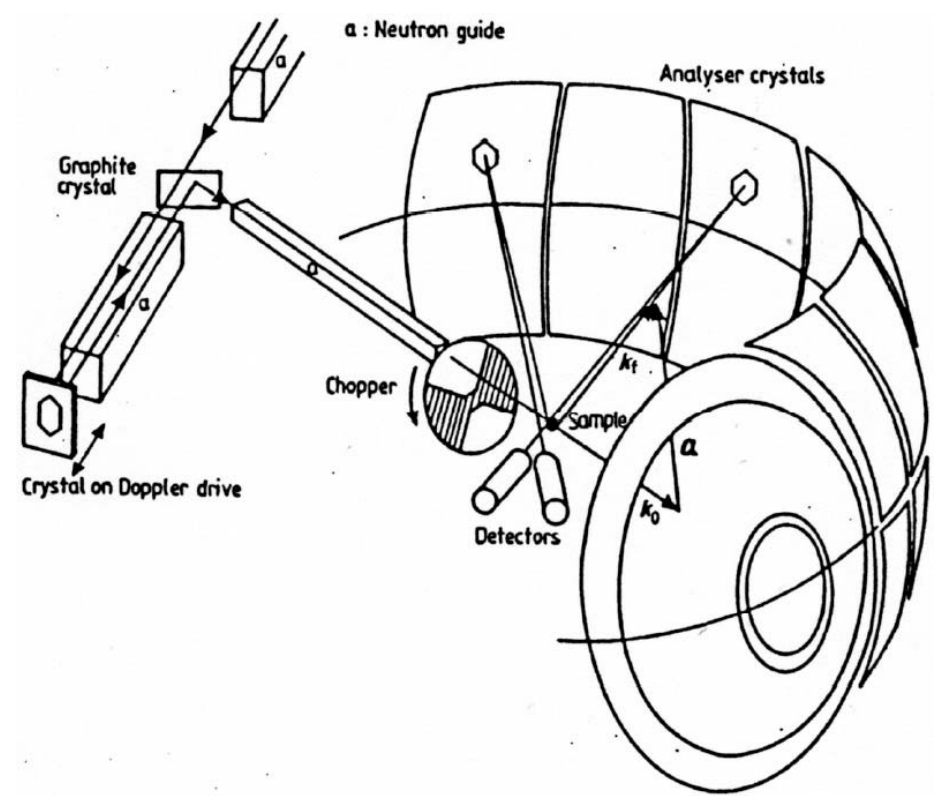

Figure 14. Spectromètre à rétrodiffusion d'après [3].

\subsubsection{Spectromètre à écho de spin (ou neutron spin echo)}

Ce sont des appareils à neutrons polarisés qui utilisent le fait que, dans un champ magnétique, les moments magnétiques associés aux spins des neutrons tournent autour de ce champ magnétique (rotation de Larmor). La mesure des vitesses avant et après le processus de diffusion se fait en étudiant le nombre de rotations de Larmor (quelques millions) que font les spins en passant dans une zone de champ magnétique: les neutrons les plus rapides restent moins longtemps, donc font moins de précessions, les neutrons les plus lents font plus de précessions. Le faisceau est rendu monochromatique par un sélecteur de vitesses, polarisé par un supermiroir et les spins sont mis en rotation autour du champ $\mathrm{B}_{\circ}$ dans un premier solénoïde (figure 15). Cependant, dû à la distribution en longueurs d'onde $\Delta \lambda$ (en vitesses $\Delta v$ ) du faisceau (grossièrement monochromatique seulement), la polarisation du faisceau se perd rapidement dans cette première zone de précession.

Au niveau de l'échantillon, un retournement des spins est effectué (diffusion avec spin flip ou flipper $\pi$ ). De ce fait, les spins qui avaient pris de l'avance dans leur rotation se retrouvent en retard, tandis que ceux qui avaient pris du retard se retrouvent en avance. Après diffusion sur l'échantillon une deuxième zone de précession, identique à la première fait précesser les spins. A la sortie de cette zone, les retards et les avances de rotation se trouvant compensées, un écho de la polarisation initiale est retrouvé. La variation de cette polarisation en fonction du champ (figure 16) dans les deux bobines (scan symétrique) représente la fonction intermédiaire $\mathrm{I}(\vec{Q}, \mathrm{t})$ du système ( $\mathrm{t}$ est proportionnel à $\mathrm{B}_{\circ}$ ). Avec le spin echo, des résolutions en énergie inférieures au neV peuvent être atteintes, donc des mouvements aussi lents que $10^{-6}$ secondes.

\subsubsection{Les domaines d'utilisation des différents instruments de diffusion inélastique}

Les domaines accessibles en transfert d'énergie et en transfert de moment (vecteur de diffusion) sont représentés sur la figure 17 pour les différents instruments de diffusion inélastique. 


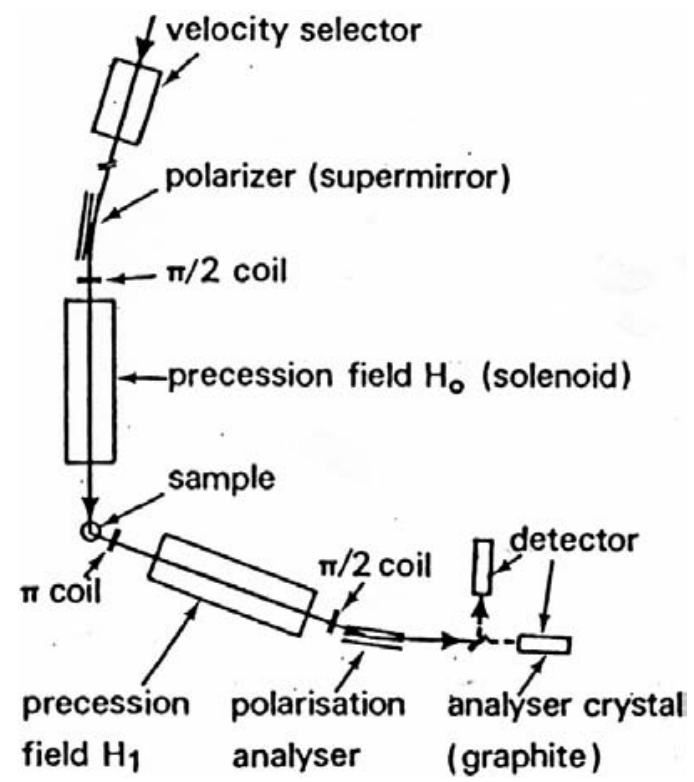

Figure 15. Spectromètre à écho de spin.



Figure 16. Variations de la polarisation en fonction du champ (scan symétrique).

\section{CE QUE L'ON VOIT : QUELQUES MODÈLES}

\subsection{Voir les structures (diffusion élastique cohérente)}

Étudier une structure c'est étudier comment sont situés les atomes les uns par rapport aux autres ou, à plus grande échelle, comment sont situés certains sous-ensembles par rapport à d'autres sous ensembles. C'est donc la diffusion cohérente qui est appropriée et la structure se manifeste par des effets d'interférences. Et dans la mesure où on parle de structure, nous allons considérer que ces atomes ou ces sous ensembles sont fixes ou, au moins, occupent une position d'équilibre autour de laquelle ils peuvent éventuellement 


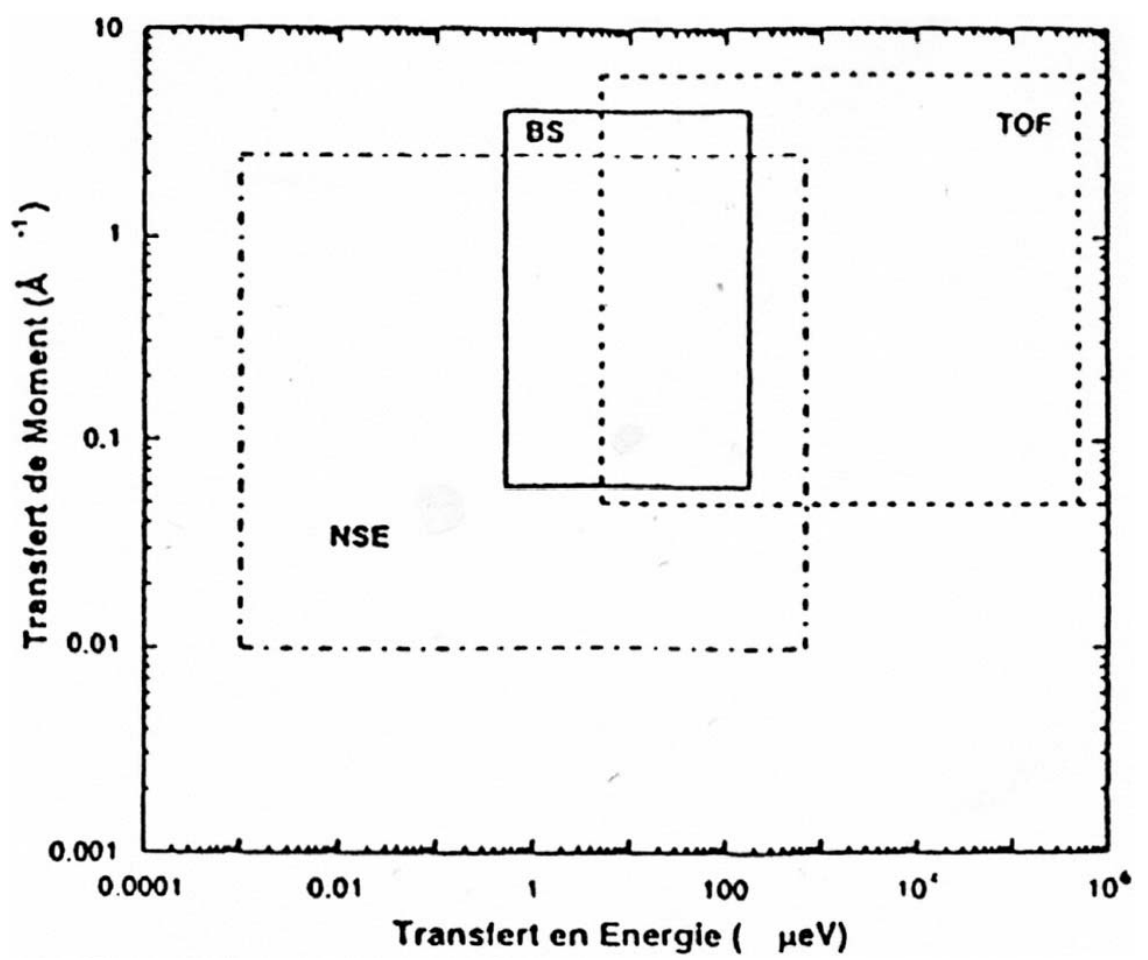

Figure 17. Les domaines accessibles en $Q$ et en $E$ pour les différents spectromètres. Les symboles sont les suivants: TOF pour temps de vol, BS pour rétrodiffusion et NSE pour écho de spin.

bouger. Nous faisons donc appel à la diffusion cohérente élastique:

$$
\left(\frac{d \sigma}{d \Omega}\right)_{c o h}^{e l}=\sum_{j j^{\prime}}\left(\vec{b}_{j} \vec{b}_{j^{\prime}} e^{-i \vec{Q} \cdot\left[\vec{R}_{j}(0)-\vec{R}_{j^{\prime}}(\infty)\right]}\right)
$$

Comme il s'agit de diffusion cohérente, dans la plupart des cas, entre l'hydrogène et le deutérium, on sera amenés à choisir le deutérium qui se voit mieux en diffusion cohérente et qui donne beaucoup moins de diffusion incohérente parasite. Pour une étude de structure, nous pouvons considérer que $\vec{R}_{j}(0)$ et $\vec{R}_{j^{\prime}}(\infty)$ sont les positions d'équilibre $\vec{R}_{j}$ et $\vec{R}_{j^{\prime}}$ des atomes j et j'. La section efficace s'écrit donc:

$$
\left(\frac{d \sigma}{d \Omega}\right)_{c o h}^{e l}=\left|\sum_{j} \bar{b}_{j} e^{i \vec{Q} \cdot \vec{R}_{j}}\right|^{2}=\mid \sum \text { Amplitudes diffusées }\left.\right|^{2}
$$

\subsubsection{Structure d'un cristal}

Commençons par regarder un cristal rigide, ayant la symétrie de translation dans les 3 directions de l'espace, et formé d'atomes qui ne bougent pas de leur position d'équilibre. Il n'y aura pas de diffusion dans l'espace réciproque, sauf pour les réflexions de Bragg $\vec{Q}=\vec{\tau}_{h k l}$ :

$$
\left(\frac{d \sigma}{d \Omega}\right)_{c o h}^{e l}=N^{2} \sum_{h k l}|F(\vec{Q})|^{2} \delta\left(\vec{Q}-\vec{\tau}_{h k l}\right)
$$


où $\mathrm{N}$ est le nombre de mailles élémentaires dans le cristal et où le facteur de structure F(hkl) est l'amplitude diffusée par tous les atomes d'une maille élémentaire:

$$
F(h k l)=\sum_{j} \bar{b}_{j} e^{2 \pi i\left(h x_{j}+k y_{j}+l z_{j}\right)}
$$

Si maintenant on considère un cristal où les atomes peuvent bouger autour de leur position d'équilibre, comme précédemment, il n’y aura de diffusion (diffraction) que sur les reflexions de Bragg, mais le facteur de structure se trouve modifié:

$$
F(h k l)=\sum_{j} \bar{b}_{j} f_{j}(Q) e^{2 \pi i\left(h x_{j}+k y_{j}+l z_{j}\right)}
$$

Chaque atome n'est plus traité comme un point de l'espace, mais comme occupant en moyenne un certain volume et $f_{j}(Q)$ est le facteur de forme de ce volume, c'est à dire, sa transformée de Fourier. Pour les vibrations thermiques, l'approximation harmonique s'applique et $f_{j}(Q)$, pour des atomes vibrant de façon isotrope, est donné par la relation:

$$
f_{j}(Q)=e^{-\frac{1}{2} Q^{2}<u_{j}^{2}>}=e^{-B_{j} \frac{\sin ^{2} \theta}{\lambda^{2}}}
$$

où l'amplitude des vibrations de l'atome $\mathrm{j}$ est représentée soit par le déplacement carré moyen $<u_{j}^{2}>$ soit par le facteur de Debye Waller $B_{j}$.

\subsubsection{Structure des objets de grande taille (grosses molécules, grains, précipités...)}

Dans la mesure où on étudie des objets dont la taille est grande par rapport aux distances interatomiques, on s'intéressera, en tout cas dans un premier temps, aux dimensions, aux formes de ces objets et à l'agencement des sous unités les unes par rapport aux autres. Dans ce cas, ce qui va intervenir en diffusion de neutrons, ce ne sont pas les atomes individuellement avec leur longueur de diffusion $\bar{b}_{j}$, mais les moyennes de ces longueurs de diffusion $\rho_{b}(r)$ par unité de volume, les densités locales de longueurs de diffusion:

$$
\rho_{b}(r)=\frac{1}{V} \int_{V} \bar{b}_{j} d^{3} r
$$

Ces moyennes sont prises sur un volume grand par rapport aux distances interatomiques, mais petit par rapport à la résolution de l'expérience. Elles s'expriment en $10^{-12} \mathrm{~cm} . \AA^{-3}$. La section efficace cohérente élastique est alors:

$$
\left(\frac{d \sigma}{d \Omega}\right)_{c o h}^{e l}=N\left|\int_{V} \rho_{b}(\vec{r}) e^{i \vec{Q} \cdot \vec{r}} d^{3} \vec{r}\right|^{2}
$$

où l'intégration se fait sur le volume $\mathrm{V}$ d'un des objet et où $\mathrm{N}$ est le nombre de ces objets.

A très petits Q, c'est à dire avec un regard qui voit grand. Lorsqu' on scrute ces objets à une échelle qui est grande devant leur dimension (petits vecteurs $\vec{Q}$, les neutrons ne voient de ces objets qu'un ensemble global, caractérisé par son rayon de giration $R_{G}$. On est alors dans le domaine de Guinier et le facteur de structure correspond au développement suivant:

$$
S(\vec{Q})=1-\frac{Q^{2} R_{G}^{2}}{3}+\cdots
$$

C'est pour des vecteurs $\vec{Q}$ qui vont au delà du domaine de Guinier que la fonction $\mathrm{S}(\mathrm{Q})$ va devenir spécifique et représenter la forme exacte des particules. 
Grands objets en solution, variation de contraste. Si les objets sont en solution, la densité qui intervient pour la diffusion est le contraste par rapport à la densité de diffusion de la solution: $\rho_{b}(\vec{r})-\rho_{b}^{\text {sol }}$.

$$
\left(\frac{d \sigma}{d \Omega}\right)_{c o h}^{e l}=N\left|\int_{V}\left[\rho_{b}(\vec{r})-\rho_{b}^{s o l}\right] e^{i \vec{Q} \cdot \vec{r}} d^{3} \vec{r}\right|^{2}
$$

Si on considère que $\rho_{b}(\vec{r})$ est la somme de deux termes: une valeur moyenne constante sur tout le volume de l'objet $\overline{\rho_{b}}$ et d'une fluctuation $\Delta \rho_{b}(\vec{r})$ autour de cette valeur moyenne:

$$
\rho_{b}(\vec{r})=\overline{\rho_{b}}+\Delta \rho_{b}(\vec{r})
$$

alors la section efficace peut s'écrire:

$$
\left(\frac{d \sigma}{d \Omega}\right)_{c o h}^{e l}=N\left|\int_{V}\left[\left(\overline{\rho_{b}}-\rho_{b}^{s o l}\right)+\Delta \rho_{b}(\vec{r})\right] e^{i \vec{Q} \cdot \vec{r}} d^{3} \vec{r}\right|^{2}
$$

On peut faire varier le contraste moyen $\left(\overline{\rho_{b}}-\rho_{b}^{\text {sol }}\right)$ en changeant les proportions d'eau légère et d'eau lourde $\left(\rho_{\mathrm{H}_{2} \mathrm{O}}^{\text {sol }}=-0.005610^{-12} \mathrm{~cm} . \AA^{-3}, \rho_{D_{2} O}^{s o l}=+0.064010^{-12} \mathrm{~cm} . \AA^{-3}\right.$. On a ainsi accès à différentes fonctions de Q qui permettent de remonter à la forme de l'objet et aux fluctuations $\Delta \rho_{b}(\vec{r})$.

\subsection{Voir la dynamique à travers les mouvements individuels d'atomes (diffusion inélastique incohérente)}

Pour étudier les dynamiques, la première approche est de regarder les corrélations temporelles qui existent entre un atome au temps 0 et ce même atome au temps t. On s'attache alors à la diffusion incohérente inélastique des neutrons, à des mouvements individuels d'atomes. Ce sont donc les atomes qui ont de grosses sections efficaces incohérentes qui sont les meilleurs candidats pour l'étude de la dynamique. Au premier rang desquels on y trouve l'hydrogène, cette fois sous sa forme protnonée et non plus deutérée.

Les instruments utilisés pour ces études sont essentiellement les spectromètres à temps de vol et, pour les hautes résolutions et les petits transferts d'énergie (mouvements lents), le spectromètre à rétrodiffusion et le spectromètre à echo de spin. Comme le montre la figure 18, on parle de "diffusion quasiélastique" si le spectre mesuré présente un élargissement du pic élastique et de "diffusion inélastique" si la diffusion est bien séparée de ce pic central élastique. Nous allons passer en revue les mouvements de translation et de rotation.

\subsubsection{Les mouvements de translation}

Les mouvements de translation se traduisent par une diffusion quasiélastique caractéristique du mouvement. Dans un liquide par exemple, l'élargissement en énergie de $S_{i n c}(\vec{Q}, \omega)$ est d'autant plus grand que le vecteur Q est grand. En effet, à petits $Q$, c'est à dire lorsque les neutrons regardent le mouvement d'un atome à grande échelle, en considérant de grands déplacements, ils voient des mouvements lents résultants des nombreux chocs que subit cet atome: l'élargissement en $\omega$ est faible. Par contre, à grands $\mathrm{Q}$, lorsque les neutrons regardent le mouvement à petite échelle, pour de petits déplacements, ils voient un atome presque libre de ses mouvements, en tout cas jusqu'à ce qu'il rencontre ses premiers voisins: le mouvement est rapide et l'élargissement en $\omega$ est important. C'est ce que montre la figure 19 pour l'argon liquide [3]. Dans les polymères, les mouvements des chaînons sont beaucoup plus lents. On peut les observer par des expériences sur un spectromètre à spin echo. C'est alors la fonction intermédiaire $\mathrm{I}_{s}(\vec{Q}, t)$ qui est mesurée et sa décroissance en $t$ représente directement la dynamique étudiée. C'est ce qu'illustre la figure 20. Pour un polymère en solution (a), la fonction $\mathrm{I}_{s}(\vec{Q}, t)$ tombe à zero selon une exponentielle étirée, plus lentement que les mouvements de diffusion habituels des atomes [5]. Par contre, pour un polymère fondu (b), cette fonction commence par décroitre, puis présente un plateau, reflétant ainsi la très lente corrélation d'un chaînon avec lui même, dans son mouvement de reptation, contraint qu'il est à l'intérieur d'un tube formé par les points d'enchevêtrement des chaînes voisines [6]. 


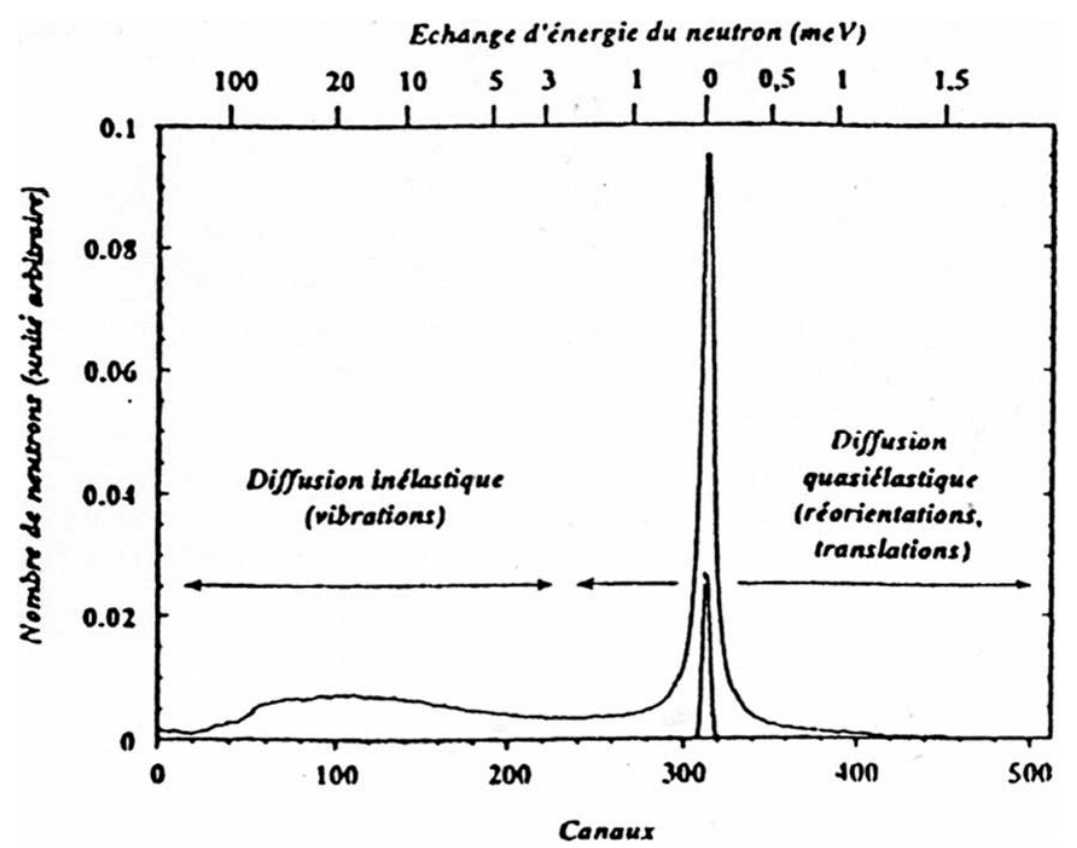

Figure 18. Diffusion quasiélastique et diffusion inélastique, d'après [4].

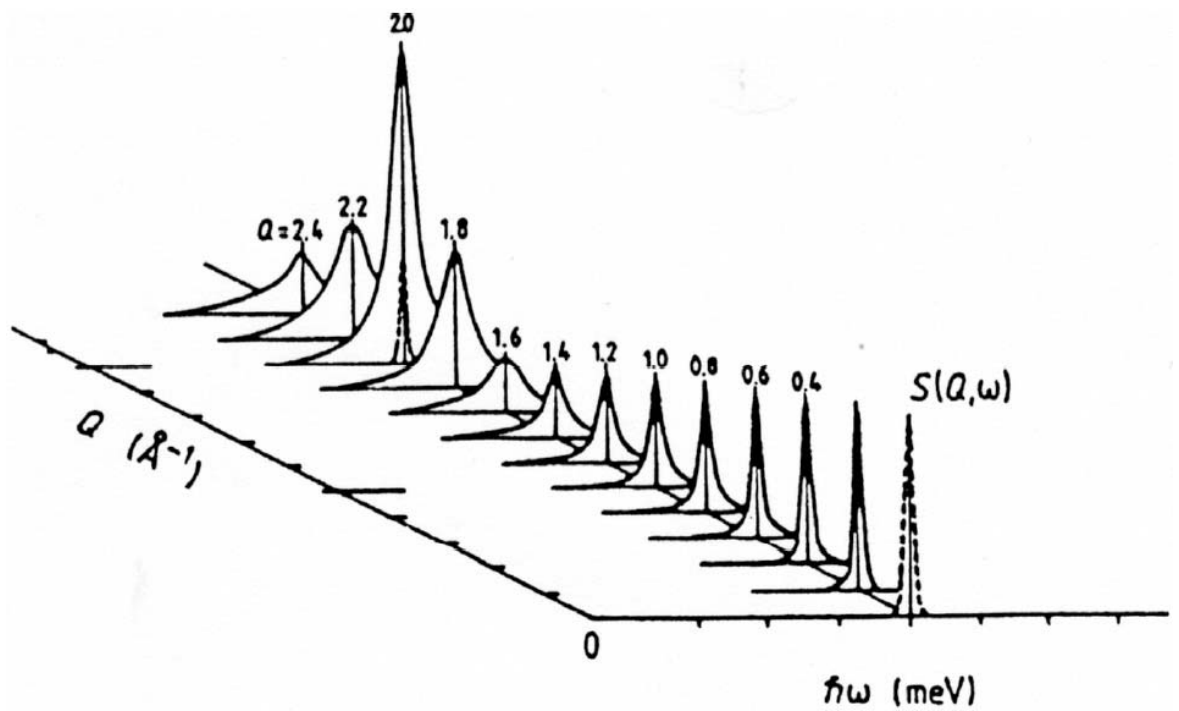

Figure 19. Dépendance en énergie de l'intensité quasi-élastique de l'argon liquide pour différents vecteurs Q.

\subsubsection{Les mouvements de rotation}

Selon les cas, les mouvements de rotation peuvent donner lieu soit à une diffusion inélastique, avec des niveaux bien définis, correspondant à des sauts d'une position à une autre position, soit à une diffusion quasiélastique qui représente une rotation continue. La figure 21 illustre, pour l'ion ammonium de $\mathrm{NH}_{4} \mathrm{ClO}_{4}$, le passage du régime de sauts par effet tunnel à basse température au régime où ces atomes tournent pratiquement librement lorsque la température s'élève [7]. 


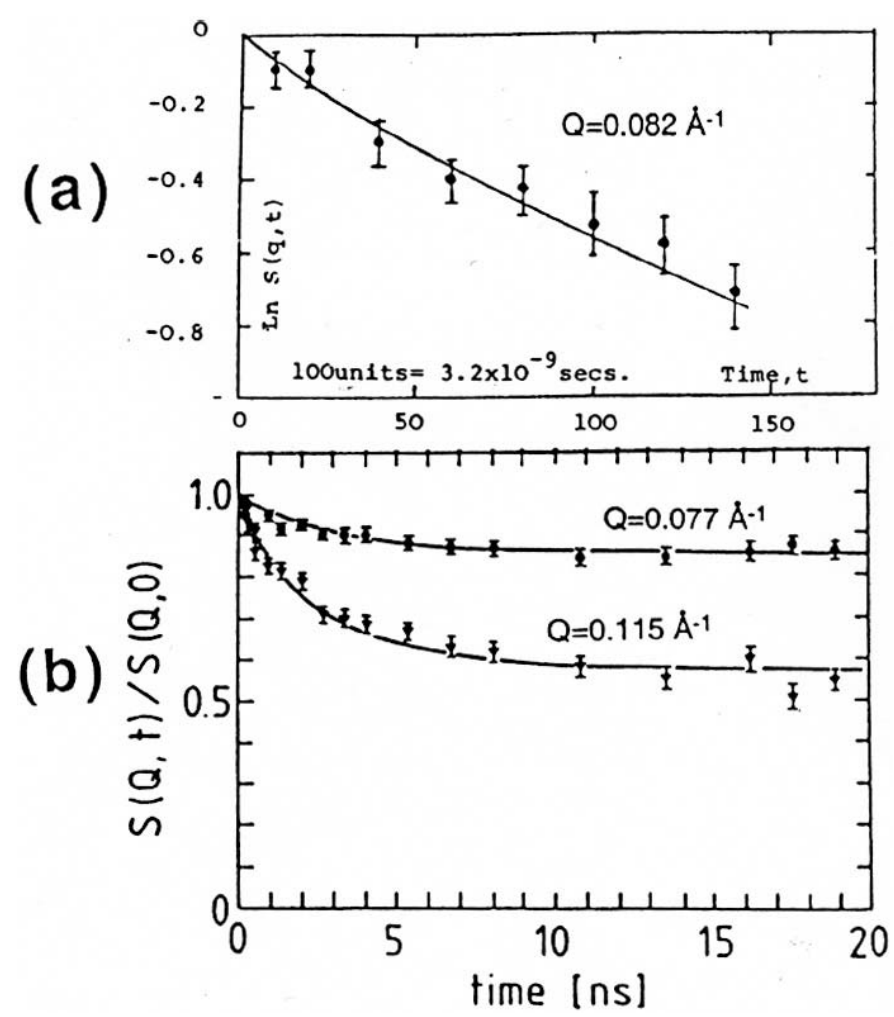

Figure 20. Fonction intermédiaire mesurée par écho de spin: (a) pour un polymère en solution, (b) pour un polymère fondu.

\subsection{Voir les cages dans lesquelles les atomes restent enfermés (diffusion élastique incohérente)}

Si on reste en diffusion incohérente (mouvements individuels d'atomes), mais qu'on se penche sur la partie élastique de la diffusion, nous mesurons les interférences entre un atome au temps zero et le même atome au temps infini:

$$
\left(\frac{d \sigma}{d \Omega}\right)_{i n c}^{e l}=\sum_{j} \overline{(\Delta b)_{j}^{2}}\left(e^{-i \vec{Q} \cdot\left[\vec{R}_{j}(0)-\vec{R}_{j}(\infty)\right]}\right)
$$

Si l'atome part au loin, il n'y a pas de corrélations donnant lieu à de la diffusion élastique incohérente. Mais si l'atome reste confiné dans une partie de l'espace, la mesure correspond à une fonction de Q qui représente la transformée de Fourier de cette partie de l'espace. Là encore, comme il s'agit d'une diffusion incohérente, l'hydrogène (noyau proton) est l'atome privilégié sur lequel se font presque toutes les expériences de ce type.

Nous allons passer en revue les différents systèmes évoqués plus haut et voir quel type de diffusion élastique incohérente on rencontre.

Pour un cristal constitué d'atomes immobiles, $\vec{R}_{j}(0)=\vec{R}_{j}(\infty)=\vec{R}_{j}$. Les atomes sont des points immobiles et il n'y a pas d'interférence: la section efficace ne dépend pas de Q. L'hydrogène diffuse de façon incohérente et élastique, avec la même intensité dans toutes les directions de l'espace. Cette diffusion est généralement considérée comme un bruit de fond parasite. 




Figure 21. Rotation des ions $\mathrm{NH}_{4}^{+}$dans $\mathrm{NH}_{4} \mathrm{ClO}_{4}$.

Pour un cristal constitué d'atomes vibrant autour de leurs positions d'équilibres. Chaque atome occupe dans sa vibration une position de l'espace et on en mesure la transformée de Fourier qu'on peut considérer comme un facteur de forme. En appliquant l'approximation harmonique, on arrive à:

$$
\left(\frac{d \sigma}{d \Omega}\right)_{i n c}^{e l}=\sum_{j} \overline{(\Delta b)_{j}^{2}} e^{-i<u^{2}>\cdot Q^{2}}=N \frac{\sigma_{i n c}}{4 \pi} e^{-<u^{2}>\cdot Q^{2}}
$$

où on retrouve le déplacement carré moyen $<u^{2}>$. La diffusion se fait toujours dans toutes les directions de l'espace, mais elle n'est plus isotrope.

Pour les rotations. la contribution élastique est appelée le facteur de structure élastique incohérent (EISF en abréviation anglaise). La figure 22a montre pour le sulfolane la séparation de la section efficace incohérente entre partie élastique et partie quasiélastique. La figure 22b compare la variation en $\mathrm{Q}$ de l'EISF mesuré avec les prédictions fournies par différents modèles de rotation de l'hydrogène dans la molécule [8].

Pour une molécule biologique. où les mouvements de certains atomes d'hydrogène tiennent à la fois de la vibration des atomes et de la libration d'une partie de la molécule, on mesurera aussi la transformée de Fourier de l'espace occupé au cours du mouvement. La formule 4.14 s'applique avec un déplacement carré moyen qui est la somme entre le déplacement carré moyen de vibration et le déplacement carré moyen de libration. 
(a)

(b)

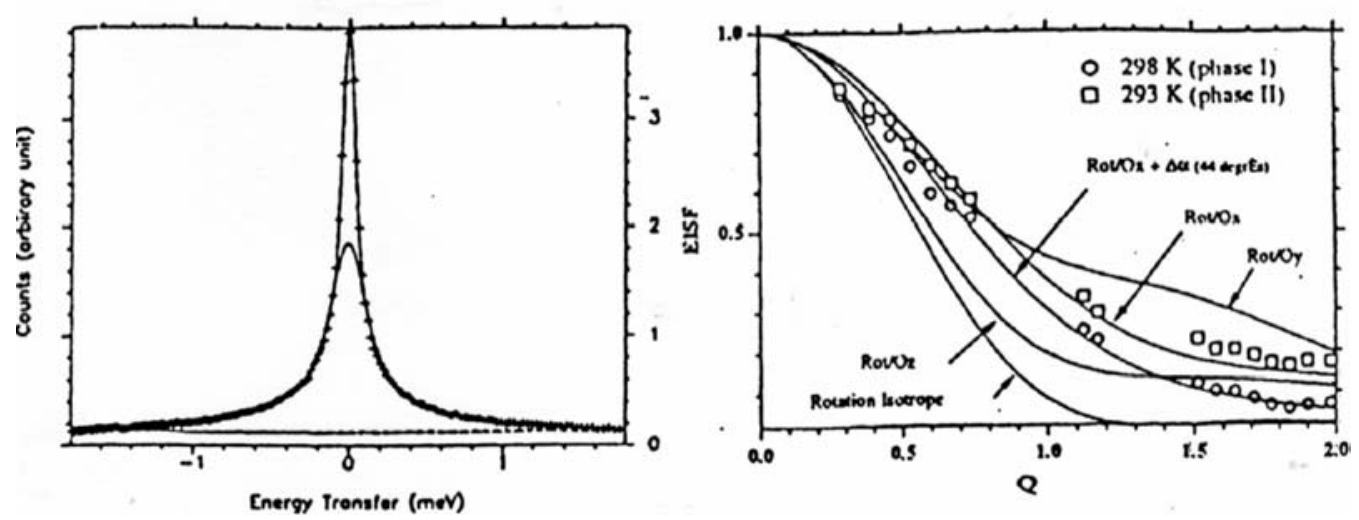

Figure 22. Diffusion par le sulfolane (a) séparation entre l'EISF et le quasi-elastique, (b) variation en Q de l'EISF.

\section{CONCLUSION}

La diffusion de neutrons a vu le jour et s'est développée par et pour les chercheurs de la matière condensée. Les biologistes n'y sont venus ensuite que petit à petit. Ils ont découvert les immenses possibilités que leur offre cette technique. Ils ont été intéressés d'abord par l'aspect structure, puis par le côté dynamique. Parallèlement, une double évolution a vu le jour: avec les grandes longueurs d'onde, des instruments se sont spécialisés pour l'étude des objets de grande dimension, et les méthodes d'analyse se sont sophistiquées pour permettre des études à haute résolution sur les grosses molécules. Aujourd'hui, les biologistes diposent d'une instrumentation de grande qualité pour la diffusion de neutrons et de méthodes bien rôdées pour mener leurs études. Ils doivent en profiter.

\section{Références}

[1] L. Koester et W.B. Yelon, Neutron Diffraction Newsletter, édité par G.H. Lander et M.H. Mueller, 1982.

[2] R. Scherm et B. Fåk, in "Neutron and Synchrotron for Condensed Matter Studies (H.E.R.C.U.L.E.S)", edité par J. Baruchel, J.L. Hodeau, M.S. Lehmann, J.R. Regnard et C. Schlenker, Volume 1, Springer-Verlag, Berlin-Heidelberg et Les Editions de Physique, Les Ulis, 1993, pp 113-143.

[3] M. Bée, in "Quasielastic Neutron Scattering, Principles and Applications in Solis State Chemistry, Biology and Materials Science", Adam Hilger, 1988.

[4] M. Bée, in "Diffusion Quasiélastique des Neutrons", Ecole Thématique de la Société Française de Neutronique, édité par M. Bée.

[5] L.K. Nicholson, J.S. Higgins et J.B. Hayter in "Neutron Spin Echo, Proceedings, Grenoble 1979", p75, Edité par F. Mezei, Springre-Verlag, Berlin, Heidelberg, New York, 1980.

[6] D. Richter, L. Wilner, A. Zirkel, B. Farago, L.J. Fetters et J.S. Huang, Phys. Rev. Let. 71 (1993) 4158.

[7] M. Prager, B. Alefeld, A. Heidemann in "Magnetic Resonance and Related Phenomena", édité par H.B. Brunner et al., Groupement Ampère, Heidelberg 1976.

[8] J. Combet, M. Bée, D. Djurado, G. D’Azenza et G. Commandeur, Physica B233 (1997) 95. 